\title{
FIRE SPECTROSCOPY OF FIVE LATE-TYPE T DWARFS DISCOVERED WITH THE WIDE-FIELD INFRARED SURVEY EXPLORER*
}

\author{
Adam J. Burgasser ${ }^{1,2,11}$, Michael C. Cushing ${ }^{3}$, J. Davy Kirkpatrick ${ }^{4}$, Christopher R. Gelino ${ }^{4}$, Roger L. Griffith ${ }^{4}$, \\ Dagny L. Looper ${ }^{5}$, Christopher Tinney ${ }^{6}$, Robert A. Simcoe ${ }^{2}$, John J. Bochanski ${ }^{7}$, Michael F. Skrutskie ${ }^{8}$, A. Mainzer ${ }^{3}$, \\ Maggie A. Thompson ${ }^{9}$, Kenneth A. Marsh $^{4}$, James M. Bauer $^{3}$, and Edward L. Wright ${ }^{10}$ \\ ${ }^{1}$ Center for Astrophysics and Space Science, University of California San Diego, La Jolla, CA 92093, USA; aburgasser@ucsd.edu \\ ${ }^{2}$ Massachusetts Institute of Technology, Kavli Institute for Astrophysics and Space Research, 77 Massachusetts Avenue, Cambridge, MA 02139, USA \\ ${ }^{3}$ NASA Jet Propulsion Laboratory, 4800 Oak Grove Drive, Pasadena, CA 91109, USA \\ ${ }^{4}$ Infrared Processing and Analysis Center, MS 100-22, California Institute of Technology, Pasadena, CA 91125, USA \\ 5 Institute for Astronomy, University of Hawaii, 2680 Woodlawn Drive, Honolulu, HI 96822, USA \\ ${ }^{6}$ Department of Astrophysics, School of Physics, University of New South Wales, NSW 2052, Australia \\ ${ }^{7}$ Department of Astronomy and Astrophysics, The Pennsylvania State University, University Park, PA 16802, USA \\ ${ }^{8}$ Department of Astronomy, University of Virginia, Charlottesville, VA, 22904, USA \\ ${ }^{9}$ The Potomac School, 1301 Potomac School Road, McLean, VA 22101, USA \\ ${ }^{10}$ Department of Physics and Astronomy, UCLA, Los Angeles, CA 90095-1562, USA \\ Received 2011 March 11; accepted 2011 April 11; published 2011 June 23
}

\begin{abstract}
We present the discovery of five late-type T dwarfs identified with the Wide-field Infrared Survey Explorer (WISE). Low-resolution near-infrared spectroscopy obtained with the Magellan Folded-port InfraRed Echellette reveal strong $\mathrm{H}_{2} \mathrm{O}$ and $\mathrm{CH}_{4}$ absorption in all five sources, and spectral indices and comparison to spectral templates indicate classifications ranging from T5.5 to T8.5: The spectrum of the latest-type source, WISE J1812+2721, is an excellent match to that of the T8.5 companion brown dwarf Wolf 940B. WISE-based spectrophotometric distance estimates place these T dwarfs at $12-13 \mathrm{pc}$ from the Sun, assuming they are single. Preliminary fits of the spectral data to the atmosphere models of Saumon \& Marley indicate effective temperatures ranging from $600 \mathrm{~K}$ to $930 \mathrm{~K}$, both cloudy and cloud-free atmospheres, and a broad range of ages and masses. In particular, two sources show evidence of both low surface gravity and cloudy atmospheres, tentatively supporting a trend noted in other young brown dwarfs and exoplanets. In contrast, the high proper motion T dwarf WISE J2018-7423 exhibits a suppressed $K$-band peak and blue spectrophotometric $J-K$ colors indicative of an old, massive brown dwarf; however, it lacks the broadened $Y$-band peak seen in metal-poor counterparts. These results illustrate the broad diversity of low-temperature brown dwarfs that will be uncovered with WISE.
\end{abstract}

Key words: brown dwarfs - stars: fundamental parameters - stars: individual (WISEPC J161705.75+180714.0, WISEPC J181210.85+272144.3, WISEPC J201824.98-742326.1, WISEPC J231336.41-803701.4, WISEPC J235941.07-733504.8) - stars: low-mass

Online-only material: color figures

\section{INTRODUCTION}

The discovery in 1995 of a faint companion to the nearby M dwarf Gliese 229 galvanized the field of brown dwarf observational astrophysics. Its near-infrared spectrum exhibits strong $\mathrm{H}_{2} \mathrm{O}$ and $\mathrm{CH}_{4}$ absorption, unambiguous indicators of a low-temperature, substellar atmosphere (Nakajima et al. 1995; Oppenheimer et al. 1995). These features now define the $\mathrm{T}$ dwarf spectral class (Burgasser et al. 2006b), the coldest known brown dwarfs with effective temperatures extending down to $T_{\text {eff }} \approx 500 \mathrm{~K}$ (e.g., Burningham et al. 2008; Lucas et al. 2010). Over $200 \mathrm{~T}$ dwarfs have been uncovered in the past 15 years ${ }^{12}$ identified primarily in wide-field, near-infrared imaging surveys such as the Two Micron All Sky Survey (2MASS; Skrutskie et al. 2006; e.g., Burgasser et al. 2002; Looper et al. 2007), the Sloan Digital Sky Survey (SDSS; York et al. 2000; e.g., Geballe et al. 2002; Chiu et al. 2006), the United Kingdom Infrared Telescope Deep Sky Survey (UKIDSS; Lawrence et al.

\footnotetext{
* This paper includes data gathered with the $6.5 \mathrm{~m}$ Magellan Telescopes located at Las Campanas Observatory, Chile.

${ }^{11}$ Hellman Fellow.

12 For an up-to-date compilation, see the DwarfArchives website, http://dwarfarchives.org.
}

2007; e.g., Lodieu et al. 2007; Burningham et al. 2010b), and the Canada-France Brown Dwarf Survey (Delorme et al. 2008a, 2008b; Reylé et al. 2010).

Efforts are now underway to identify even colder brown dwarfs, sources whose atmospheres are anticipated to bridge the temperature gap between the known population and the Jovian planets $\left(T_{\text {eff }} \lesssim 125 \mathrm{~K}\right)$. This is the realm in which directly detectable young extrasolar planets are now being found and investigated (e.g., Kalas et al. 2008; Marois et al. 2008; Janson et al. 2010; Bowler et al. 2010b; Currie et al. 2010). Theoretical models of substellar atmospheres predict several interesting chemical transitions at these temperatures, including the emergence of $\mathrm{NH}_{3}$ as a prominent absorber at near-infrared wavelengths; the condensation of $\mathrm{H}_{2} \mathrm{O}$ and formation of thick ice clouds; and the condensation of alkali salts, depleting brown dwarf atmospheres of spectrally prominent $\mathrm{K}$ I and Na I gases (Lodders 1999; Lodders \& Fegley 2002; Marley et al. 2002; Burrows et al. 2003; Saumon et al. 2006; Leggett et al. 2007). All of these transitions have been suggested as possible triggers for the definition of a new spectral class, tentatively designated the Y-dwarf class (Kirkpatrick 2005). These "ultracold" brown dwarfs may also comprise the bulk of the Galactic substellar population. Depending on the underlying mass function, number 


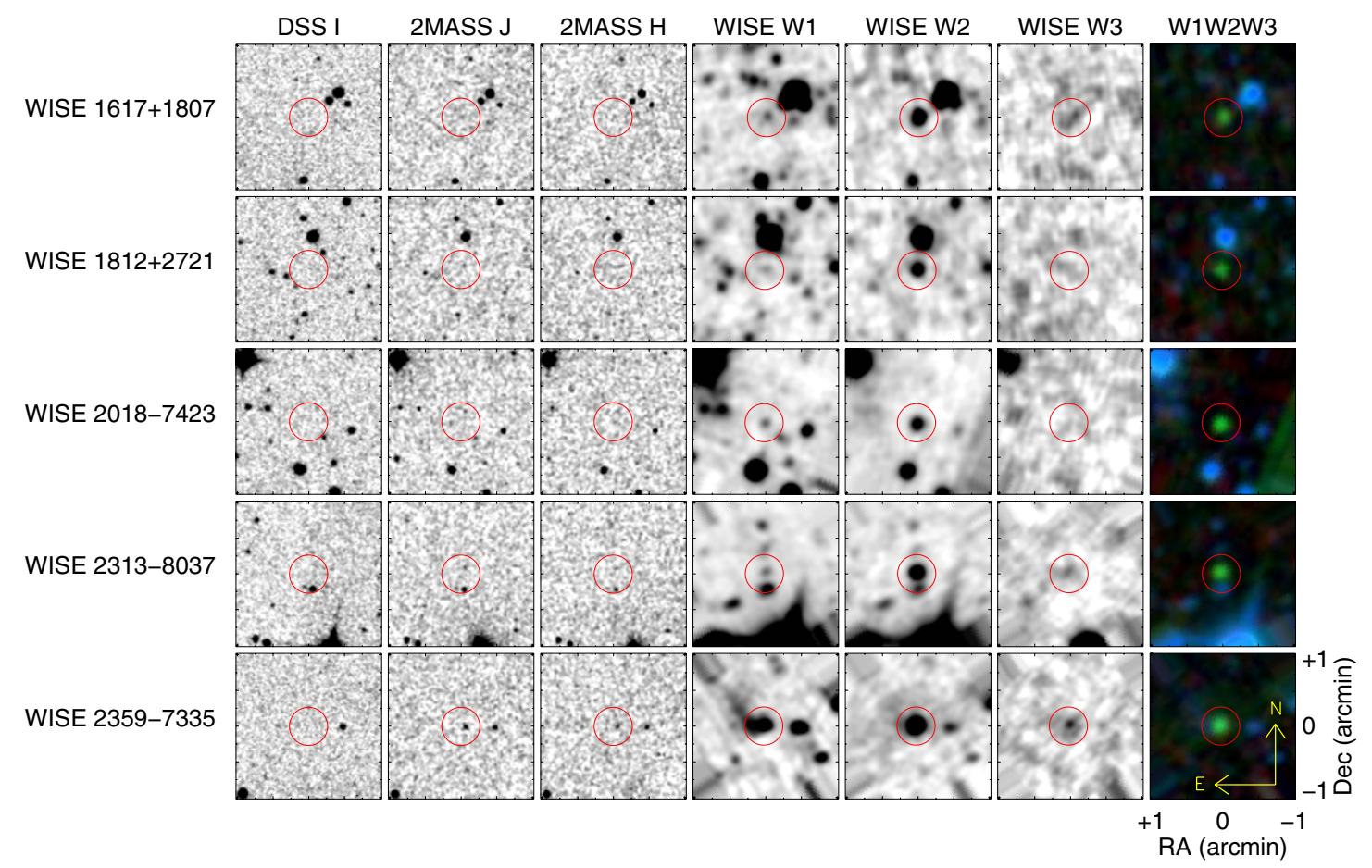

Figure 1. Finderchart images of the five WISE T dwarfs, showing $2^{\prime} \times 2^{\prime}$ fields oriented with north up and east toward the left. DSS $I_{N}$, 2MASS $J H$, and $W I S E$ W1W2W3 images are centered on the WISE coordinates for each source (red circle). The rightmost image shows a false color composite of the three WISE images, with blue, green, and red represented by $W 1, W 2$, and $W 3$, respectively.

(A color version of this figure is available in the online journal.)

densities for brown dwarfs colder than $600 \mathrm{~K}$ may exceed those of their warmer counterparts by a factor of a few or more (Burgasser 2004; Saumon \& Marley 2008). These sources would also sample the minimum brown dwarf formation mass, an important statistic for brown dwarf formation theories (e.g., Whitworth \& Stamatellos 2006), and a determinant for the total baryonic mass associated with compact objects (e.g., Graff \& Freese 1996).

Two intriguing sources-both companions to nearby stars-have recently been identified whose estimated $T_{\text {eff }} \approx$ 300-400 K may broach the Y-dwarf regime (Luhman et al. 2011; Liu et al. 2011). However, their extremely faint nearinfrared magnitudes $(J \gtrsim 21.5)$ have so far impeded spectroscopic follow-up. In order to identify a significant sample of similarly cold brown dwarfs, search programs must shift to mid-infrared wavelengths where the majority of spectral flux emerges (Burrows et al. 2003). The Wide-field Infrared Survey Explorer (WISE; Wright et al. 2010), which has surveyed the full sky in four infrared bands centered at wavelengths of $3.4 \mu \mathrm{m}$ (W1), $4.6 \mu \mathrm{m}$ (W2), $12 \mu \mathrm{m}$ (W3), and $22 \mu \mathrm{m}$ (W4), provides an opportunity to find these cold brown dwarfs. The $W 1$ and $W 2$ bands were specifically designed to differentiate $\mathrm{T}$ dwarfs from background sources, sampling the strong $3.3 \mu \mathrm{m} \mathrm{CH}$ band and the pseudocontinuum peak at $4.6 \mu \mathrm{m}$, respectively (Oppenheimer et al. 1998; Burrows et al. 2003; Golimowski et al. 2004). Mainzer et al. (2011) have recently reported the first cold brown dwarf discovery with WISE, WISEPC J045853.90+643451.9 (hereafter WISE J0458+6434), a source which exhibits nearly saturated near-infrared $\mathrm{H}_{2} \mathrm{O}$ and $\mathrm{CH}_{4}$ bands consistent with a $\sim$ T9 classification (see also Gelino et al. 2011).

In this article, we report the discovery of five new late-type $\mathrm{T}$ dwarfs identified in WISE and confirmed through nearinfrared spectroscopy with the Folded-port Infrared Echellette
(FIRE; Simcoe et al. 2008, 2010). In Section 2, we describe the selection of these sources based on WISE photometry and additional survey data. In Section 3, we describe our followup imaging and spectroscopic observations that confirm the T dwarf nature of these sources. In Section 4, we derive spectral classifications using both spectral indices and comparison to near-infrared spectral templates, and estimate distances and kinematics. In Section 5, we provide additional constraints on the atmospheric and physical properties of these sources through spectral model fits using the calculations of Saumon \& Marley (2008). In Section 6, we discuss the properties of individual discoveries in detail. Results are summarized in Section 7.

\section{CANDIDATE SELECTION}

\subsection{WISE Photometry}

Candidate late-type $\mathrm{T}$ dwarfs were selected from the WISE coadd source working database, as described in detail in Mainzer et al. (2011) and J. D. Kirkpatrick et al. (2011, in preparation). In brief, sources were selected to have $W 1-W 2 \geqslant 2, W 2-$ $W 3 \leqslant 2.5$ (to exclude extragalactic sources; see Wright et al. 2010), a $W 2$ signal-to-noise ratio $\geqslant 10$, and a point-spread function consistent with an unresolved point source. These sources were then compared to optical and near-infrared imaging survey data from the Digitized Sky Survey (DSS), SDSS, and 2MASS to exclude optical counterparts and other contaminants. The five new $\mathrm{T}$ dwarfs presented here represent only a subset of the full candidate pool currently under investigation. Their designations ${ }^{13}$ and measured photometry (excluding $W 4$ ) are listed in Table 1. Figure 1 displays DSS, 2MASS, and WISE images of the fields around each target.

\footnotetext{
13 Throughout the text, we use the shorthand notation WISE Jhhmm \pm ddmm to refer to WISE sources, where the suffix is the sexagesimal right ascension (hour and minute) and declination (degree and arcminute).
} 
Table 1

Photometric Data for WISE Brown Dwarf Discoveries

\begin{tabular}{|c|c|c|c|c|c|c|c|c|c|c|}
\hline Designation & $\begin{array}{c}Y \\
(\mathrm{mag})\end{array}$ & $\begin{array}{c}J \\
(\mathrm{mag}) \\
\end{array}$ & $\begin{array}{c}H \\
(\mathrm{mag})\end{array}$ & $\begin{array}{c}K_{S} \\
(\mathrm{mag}) \\
\end{array}$ & $\begin{array}{c}W 1 \\
(\mathrm{mag})\end{array}$ & $\begin{array}{c}W 2 \\
(\mathrm{mag})\end{array}$ & $\begin{array}{c}W 3 \\
\text { (mag) }\end{array}$ & $\begin{array}{c}H-W 2 \\
(\mathrm{mag})\end{array}$ & $\begin{array}{c}W 1-W 2 \\
(\mathrm{mag})\end{array}$ & $W 2-W 3$ \\
\hline WISEPC J161705.75+180714.0 & $18.71 \pm 0.04$ & $17.66 \pm 0.08$ & $18.23 \pm 0.08$ & $\cdots$ & $16.89 \pm 0.16$ & $14.03 \pm 0.06$ & $12.48 \pm 0.48$ & $4.20 \pm 0.10$ & $2.86 \pm 0.17$ & $1.55 \pm 0.48$ \\
\hline WISEPC J181210.85+272144.3 & $\ldots$ & $18.19 \pm 0.06$ & $18.83 \pm 0.16$ & $\ldots$ & $17.32 \pm 0.21$ & $14.15 \pm 0.05$ & $>12.4$ & $4.68 \pm 0.17$ & $3.17 \pm 0.21$ & $<1.8$ \\
\hline WISEPC J201824.98-742326.1 & $\cdots$ & $17.11 \pm 0.21^{\mathrm{a}}$ & $>16.5^{\mathrm{a}}$ & $>16.6^{\mathrm{a}}$ & $16.55 \pm 0.10$ & $13.76 \pm 0.03$ & $>12.3$ & $>2.7$ & $2.80 \pm 0.11$ & $<1.5$ \\
\hline WISEPC J231336.41-803701.4 & $\ldots$ & $16.97 \pm 0.24^{\mathrm{a}}$ & $>16.2^{\mathrm{a}}$ & $>16.4^{\mathrm{a}}$ & $16.29 \pm 0.07$ & $13.77 \pm 0.04$ & $12.52 \pm 0.32$ & $>2.4$ & $2.52 \pm 0.08$ & $1.25 \pm 0.33$ \\
\hline WISEPC J235941.07-733504.8 & $\ldots$ & $16.17 \pm 0.04^{\mathrm{a}}$ & $16.07 \pm 0.07^{\mathrm{a}}$ & $16.05 \pm 0.13^{\mathrm{a}}$ & $15.12 \pm 0.04$ & $13.26 \pm 0.04$ & $11.63 \pm 0.20$ & $2.65 \pm 0.19$ & $1.86 \pm 0.06$ & $1.63 \pm 0.20$ \\
\hline
\end{tabular}

Note. ${ }^{a}$ Photometry from the 2MASS Reject Table (WISE J2018-7423 and WISE J2313-8037) and $6 \times$ Catalogs (WISE J2359-7335; Skrutskie et al. 2006). 
Table 2

Proper Motions for WISE T Dwarfs Detected in 2MASS

\begin{tabular}{|c|c|c|c|c|c|c|}
\hline \multirow[t]{2}{*}{ Source } & \multicolumn{2}{|c|}{ Astrometry } & \multirow{2}{*}{$\begin{array}{l}\Delta t \\
(\mathrm{yr})\end{array}$} & \multirow{2}{*}{ 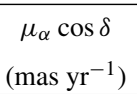 } & \multirow{2}{*}{ 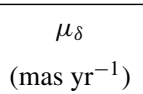 } & \multirow{2}{*}{$\begin{array}{c}V_{\tan ^{\mathrm{a}}} \\
\left(\mathrm{km} \mathrm{s}^{-1}\right)\end{array}$} \\
\hline & 2MASS & WISE & & & & \\
\hline WISE J2018-7423 & $20: 18: 24.24-74: 23: 17.92$ & $20: 18: 24.98-74: 23: 26.14$ & 9.6 & $311 \pm 32$ & $-852 \pm 31$ & $56 \pm 6$ \\
\hline WISE J2313-8037 & $23: 13: 35.37-80: 36: 56.24$ & $23: 13: 36.40-80: 37: 01.40$ & 10.4 & $242 \pm 32$ & $-496 \pm 29$ & $31 \pm 5$ \\
\hline WISE J2359-7335 & $23: 59: 40.33-73: 35: 05.33$ & $23: 59: 41.07-73: 35: 04.87$ & 9.5 & $332 \pm 26$ & $49 \pm 23$ & $20 \pm 3$ \\
\hline
\end{tabular}

Note. ${ }^{\text {a }}$ Based on the spectrophotometric distance estimates listed in Table 5.

\subsection{Additional Survey Photometry and Astrometry}

All of the WISE targets were cross-matched to the 2MASS, SDSS, and UKIDSS catalogs. One source, WISE J2359-7335, had a counterpart in the 2MASS Point Source and $6 \times$ catalogs, ${ }^{14}$ with a $J$ magnitude in the latter of $16.17 \pm 0.04$. WISE J2018-7423 and WISE J2313-8037 had faint counterparts in the 2MASS Reject Catalog, with $J=17.11 \pm 0.21$ and $16.97 \pm$ 0.24 mag, respectively (detection grades of "C" and "E"), located $\sim 7 "-10^{\prime \prime}$ from their WISE positions. We confirmed these counterparts were associated using our $J$-band FIRE acquisition images (see Section 3.2.1). WISE J1617+1807 has a nearby counterpart in the SDSS Data Release 7 catalog 9" from its WISE position, but this match appears spurious based on the source's blue optical colors $(i=21.91 \pm 0.16, i-z<-0.6)$. No common proper motion companions were found within $5^{\prime}$ of any of the WISE sources in SIMBAD or in the US Naval Observatory CCD Astrograph Catalog (Zacharias et al. 2010).

The roughly 10 year baseline between the 2MASS and WISE detections of WISE J2018-7423, WISE J2313-8037, and WISE J2359-7335 allows proper motion measurements for these sources. Astrometry from the two catalogs are listed in Table 2, and the computed proper motions incorporate uncertainties in the 2MASS and WISE positions but do not account for parallactic motion. We note that an error in the astrometric calibration pipeline of the WISE working database (now corrected) leads to an occasional large offset (of order 1") in declination coordinate. As such, these proper motions should be considered preliminary until the WISE Final Release catalog astrometry is available. Nevertheless, the relatively large angular motions of these sources, as high as $0^{\prime \prime} \cdot 91 \pm 0^{\prime \prime} \cdot 03 \mathrm{yr}^{-1}$ for WISE J2018-7423, are typical for nearby field dwarfs.

\section{OBSERVATIONS}

\subsection{Imaging}

\subsubsection{SOAR/SpartanIRC}

$J H$-band photometry of WISE J1617+1807 were obtained on 2010 March 21 (UT) in clear conditions with the Spartan Infrared Camera (SpartanIRC; Loh et al. 2004) on the $4.1 \mathrm{~m}$ SOAR telescope. The source was observed at an airmass of 1.53 with five $60 \mathrm{~s}$ exposures in each filter, dithered in $40^{\prime \prime}$ offsets. Imaging data were reduced using custom routines that perform flat fielding and sky subtraction, the latter from a sky frame created from a median stack of the dither image sequence. A $2^{\prime} \times 2^{\prime}$ mosaic was created by stacking the reduced images to a common center and averaging. Aperture photometry was measured for all sources in the mosaic, and photometric calibration was done on the 2MASS system using three bright stars in the field of view, with a zero-point uncertainty estimated from the standard deviation of photometric offsets for these three stars. Measurements are listed in Table 1.

\subsubsection{Fan Mountain/FanCam}

$Y$-band photometry of WISE J1617+1807 was obtained on 2010 April 1 (UT) in photometric conditions with the FanCam near-infrared imager mounted on the $0.8 \mathrm{~m}$ Fan Mountain telescope (Kanneganti et al. 2009). Imaging data were obtained and reduced as described in Mainzer et al. (2011), with 15 exposures of $60 \mathrm{~s}$ and 80 exposures of $30 \mathrm{~s}$ obtained in a $15^{\prime \prime}$ dither pattern, for a total exposure time of 55 minutes. Aperture photometry on the mosaicked frame was measured using standard IRAF routines, with an instrumental zero point derived by estimating $Y$-band magnitudes of neighboring stars from their 2MASS $J$ and $K_{s}$ photometry and the transformation of Hamuy et al. (2006). We found $Y=18.71 \pm 0.04$ for WISE $\mathrm{J} 1617+1807$, implying $Y-W 2=4.68 \pm 0.07$, about $0.7 \mathrm{mag}$ bluer than WISE J0458+6434 (Mainzer et al. 2011).

\subsubsection{Palomar/WIRC}

$J H$-band photometry of WISE J1812+2721 were obtained on 2010 Aug 30 (UT) using the Wide-Field Infrared Camera (WIRC; Wilson et al. 2003) mounted on the 200 inch Hale Telescope at Palomar Observatory. WIRC has a pixel scale of $0^{\prime \prime} .2487 \mathrm{pixel}^{-1}$ and a total field of view of 8.7 $\times 8^{\prime} .7$. Conditions were clear during the observations, but with high humidity and poor seeing $(\sim 2$ '.5 at $J)$. For each filter, a series of 15 exposures of $60 \mathrm{~s}$ each were obtained, dithering by $50^{\prime \prime}-100^{\prime \prime}$. Targets were observed over an airmass range of 1.09-1.13.

The imaging data were reduced using a suite of $\mathrm{IRAF}^{15}$ and FORTRAN programs provided by T. Jarrett. These routines first linearize and dark subtract the images, then create a sky frame and flat-field images for each dither set which are subtracted from and divided into (respectively) each science image. At this stage, WIRC images still contain a significant bias that is not removed by the flat field. Comparison of 2MASS and WIRC photometric differences across the array shows that this flux bias has a level of $\approx 10 \%$ and the pattern is roughly the same for all filters. Using these 2MASS-WIRC differences for many fields, we created a flux bias correction image that was applied to each of the "reduced" images. Finally, we determined an astrometric calibration for the images using 2MASS stars in the field, and the images were mosaicked together. This final mosaic was photometrically calibrated using 2MASS stars and magnitudes computed using aperture photometry. Measurements are listed in Table 1.

\footnotetext{
15 Image Reduction and Analysis Facility (IRAF; Tody 1986).
} 
Table 3

Spectroscopic Observations

\begin{tabular}{|c|c|c|c|c|c|c|}
\hline Source & Instrument & UT Date & $\begin{array}{c}\text { Integration } \\
\text { (s) }\end{array}$ & Airmass & $\begin{array}{c}\text { Calibrator } \\
\text { Star }\end{array}$ & $\begin{array}{c}\text { Conditions/ } \\
\text { Seeing }\end{array}$ \\
\hline WISE J1617+1807 & Magellan/FIRE & 2010 Apr 7 & 282 & 1.74 & $\mathrm{BD}+293523$ & Clear, 0.' 6 \\
\hline WISE J1812+2721 & Magellan/FIRE & 2010 Sep 19 & 1045 & $1.88-2.03$ & $\mathrm{BD}+303488$ & Clear, $1^{\prime \prime} 5-2^{\prime \prime}$ \\
\hline WISE J2018-7423 & Magellan/FIRE & 2010 Sep 20 & 526 & $1.53-1.55$ & HD 189588 & Clear, 0.'4 \\
\hline WISE J2313-8037 & Magellan/FIRE & 2010 Sep 20 & 526 & 1.61 & HD 189588 & Clear, $0 . .4$ \\
\hline \multirow[t]{4}{*}{ WISE J2359-7335 } & Magellan/LDSS-3 & 2005 Dec 2 & 3600 & $1.62-1.81$ & HD 10991 & Clear, $0 . .8$ \\
\hline & AAT/IRIS2 & 2006 May 15 & $600 / 600^{\mathrm{a}}$ & $1.49 / 1.60^{\mathrm{a}}$ & HIP 118079 & Clear and humid, $1^{\prime \prime} .5$ \\
\hline & AAT/IRIS2 & 2006 Jun 11 & 600 & 1.52 & HIP 118079 & Clear and humid, $2^{\prime \prime}$ \\
\hline & Magellan/FIRE & 2010 Dec 24 & 846 & $2.02-2.14$ & HD 189588 & Clear, 0.8 \\
\hline Wolf 940B & Magellan/FIRE & 2010 Sep 19 & 1045 & $1.14-1.15$ & HD 208368 & Clear, $1^{\prime \prime} .5-2^{\prime \prime}$ \\
\hline
\end{tabular}

Note. ${ }^{\text {a }}$ For $J_{l}$ and $H_{s}$ observations, respectively.

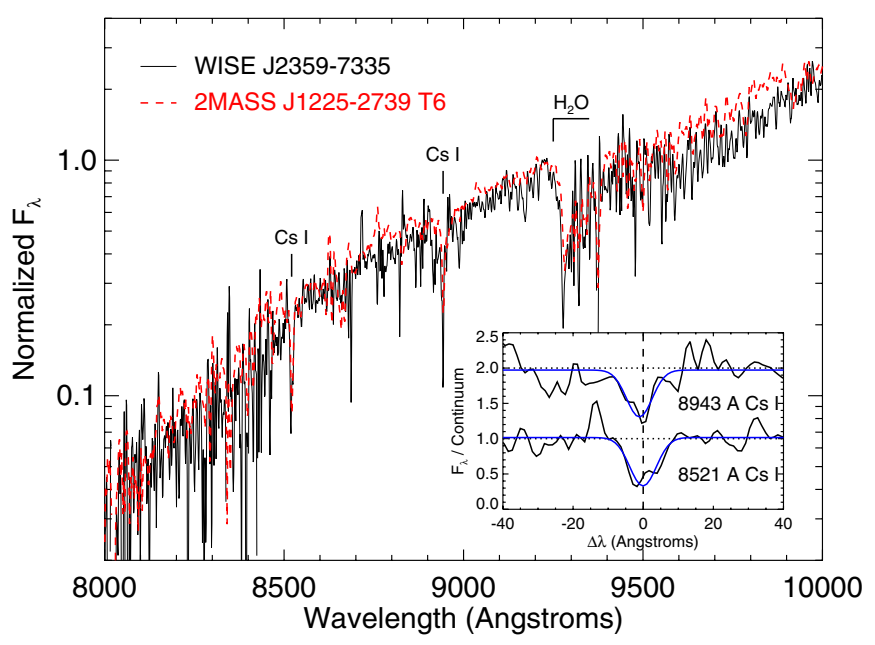

Figure 2. LDSS-3 optical spectrum of WISE J2359-7335 (black line), compared to the T6 dwarf 2MASS J1225-2739 (red line; data from Burgasser et al. 2003a). Both spectra are normalized at $9250 \AA$. Absorption features from $\mathrm{Cs} \mathrm{I}$ and $\mathrm{H}_{2} \mathrm{O}$ are indicated. The inset box shows a close-up of the $\mathrm{Cs}$ I line profiles after dividing through by the local continuum. Blue lines indicate Gaussian fits to these profiles. The $8943 \AA$ line profile in the inset is vertically offset by a constant for clarity.

(A color version of this figure is available in the online journal.)

\subsection{Spectroscopy}

\subsubsection{Magellan/LDSS-3}

Optical spectroscopy of WISE J2359-7335 was obtained on 2005 December 2 (UT) in clear conditions using the Low Dispersion Survey Spectrograph (LDSS-3; Allington-Smith et al. 1994) mounted on the Magellan $6.5 \mathrm{~m}$ Clay Telescope (see Table 3 for the complete observing log). Data were obtained using the VPH-red grism (660 lines $\mathrm{mm}^{-1}$ ) and the $0^{\prime \prime} 75$ (4 pixel) wide long slit, aligned along the parallactic angle, providing $6050-10500 \AA$ spectroscopy with $\lambda / \Delta \lambda \approx 1800$. The OG590 longpass filter was used to eliminate second order light shortward of $6000 \AA$. Two exposures of 1800 s each were obtained over an airmass range of 1.62-1.81. This was followed by observation of the nearby G2 V star HD $10991(V=9.38)$ for telluric absorption correction. HeNeAr arc lamp and flatfield quartz lamp exposures reflected off of the Clay secondary flat-field screen were obtained for dispersion and pixel response calibration. The data were reduced using the IRAF onedspec package as described in Burgasser et al. (2007).

Figure 2 displays a portion of the WISE J2359-7335 LDSS-3 spectra spanning 8000-10000 A, compared to equiv- alent data ${ }^{16}$ for the T6 dwarf 2MASS J12255432-2739466 (hereafter 2MASS J1225-2739; Burgasser et al. 1999, 2003a). Both spectra are logarithmically scaled to highlight absorption features within their steep red optical slopes. Absorption from Cs I (8521 and $8943 \AA$ ) and $\mathrm{H}_{2} \mathrm{O}$ (9250 $\AA$ bandhead) are visible. The pseudoequivalent widths of the $\mathrm{Cs}$ I lines were measured to be $4.9 \pm 1.7 \AA$ and $8.9 \pm 2.5 \AA$, respectively, the latter consistent with measurements for mid-type T dwarfs (Burgasser et al. 2003a).

\subsubsection{AAT/IRIS2}

Near-infrared spectroscopy of WISE J2359-7335 was obtained on 2006 May 15 and 2006 June 11 (UT) with the Infrared Imager and Spectrograph (IRIS2; Tinney et al. 2004) mounted on the $3.9 \mathrm{~m}$ Anglo-Australian Telescope (AAT). Conditions on both nights were clear but humid with poor seeing $\left(1^{\prime \prime} .5-2^{\prime \prime}\right)$. Spectra in the $J$ band $(1.47-1.81 \mu \mathrm{m})$ were obtained in May using the $1^{\prime \prime}$ wide slit, Sapphire-240 transmission grating and $\mathrm{J}_{l}$ filter, at an airmass of 1.49. Spectra in the $H$ band $(1.47-1.81 \mu \mathrm{m})$ were obtained in both May and June using the $1^{\prime \prime}$ wide slit, SAPPHIRE-316 transmission grating and $\mathrm{H}_{s}$ filter, at airmasses of 1.60 and 1.52 , respectively. Average resolution of these spectral modes is $\lambda / \Delta \lambda=2100$. Individual exposures of $150 \mathrm{~s}$ were obtained in ABBA dither patterns nodding along the slit, for a total exposures of $600 \mathrm{~s}$ at $J$ and $1200 \mathrm{~s}$ at $H$. The G0 V star HIP 118079 was observed on both nights immediately after the WISE target for telluric absorption and flux calibration. Exposures of Quartz halogen and Xe lamps reflected off of the AAT flat-field screen were also obtained at the beginning of each night for pixel response correction and high-order dispersion calibration of the wavelength scale. The latter calibration was then updated using the telluric $\mathrm{OH}$ emission in the science frames. Data were reduced following the procedures described in Tinney et al. (2005).

Figure 3 displays the resulting spectra, smoothed to $\lambda / \Delta \lambda=$ 500. The data have relatively low signal to noise $(\sim 5$ at the $1.27 \mu \mathrm{m}$ peak; $\sim 10$ at the $1.58 \mu \mathrm{m}$ peak), but are nevertheless sufficient to resolve the strong near-infrared $\mathrm{H}_{2} \mathrm{O}$ and $\mathrm{CH}_{4}$ bands characteristic of mid- to late-type $\mathrm{T}$ dwarf spectra.

\subsubsection{Magellan/FIRE}

Near-infrared spectroscopy of the WISE candidates and the T8.5 dwarf companion brown dwarf Wolf 940B (Burningham et al. 2009) was obtained on three separate runs during 2010

16 These data were obtained with the Low-Resolution Imaging Spectrometer (LRIS; Oke et al. 1995). 

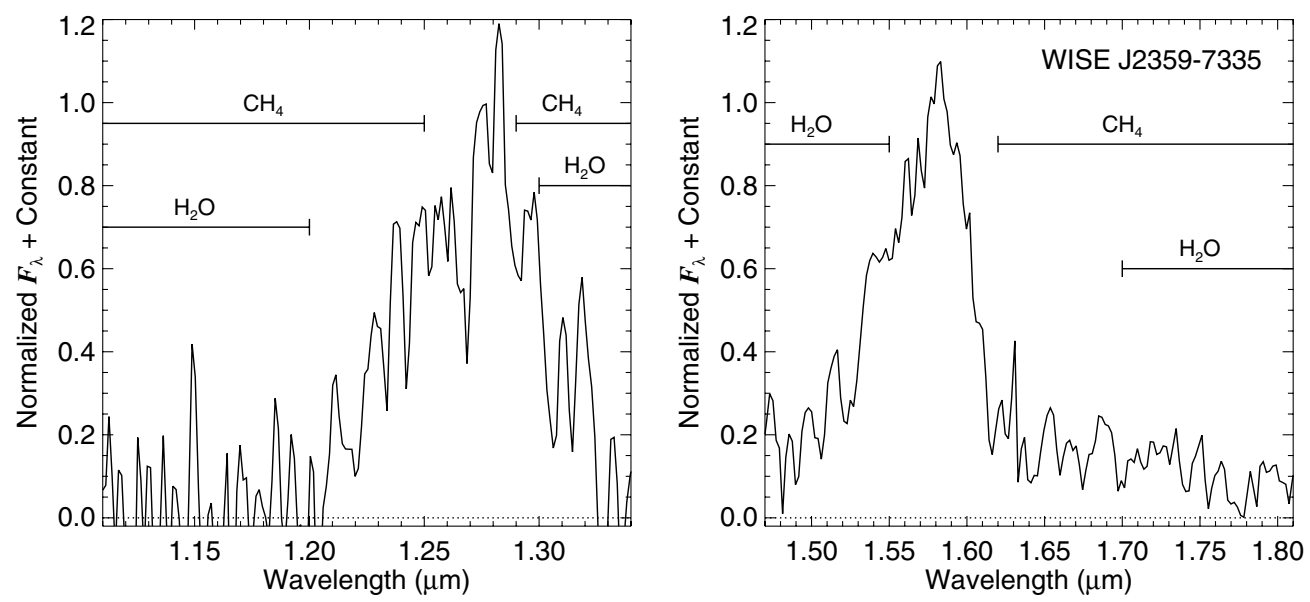

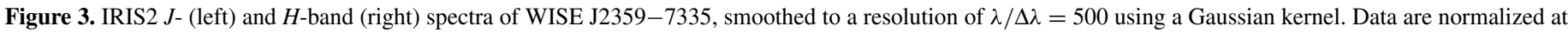
their respective flux peaks. Primary absorption bands from $\mathrm{H}_{2} \mathrm{O}$ and $\mathrm{CH}_{4}$ are indicated.

April 7, 2010 September 19-20, and 2010 December 24 (UT). All targets were observed with FIRE in its low-resolution, prism-dispersed mode, which delivers $0.85-2.45 \mu$ m continuous spectroscopy in a single order. Each source was initially acquired using FIRE's $J$-band acquisition camera, then placed into a $0^{\prime \prime} 6$ slit aligned to the parallactic angle (rotator angle 89.5). This prism/slit combination provides a variable resolution of $\lambda / \Delta \lambda=250-350$ across the near-infrared band. A series of $\mathrm{AB}$ or $\mathrm{ABBA}$ dither exposure sequences were obtained with integrations ranging from $60 \mathrm{~s}$ to $120 \mathrm{~s}$ per exposure (plus $10.6 \mathrm{~s}$ read time), the latter being the maximum permitted to avoid saturating $\mathrm{OH}$ telluric lines in the $H$ band. The spectrograph detector was read out using the four amplifier mode at "high gain" (1.2 counts/ $\mathrm{e}^{-}$) with either Fowler-8 sampling (April and September) or Sample Up The Ramp (December) modes. Each science target observation was accompanied by an observation of a nearby A0 V calibrator star (typically with $V=10-12$ ) at a similar airmass. Given FIRE's high sensitivity, these calibrators were occasionally observed out of focus and/or offset from the slit to avoid saturation in FIRE's minimum readout time (11.6 s). We also obtained exposures of a variable voltage quartz flat-field lamp (set at $1.2 \mathrm{~V}$ and $2.2 \mathrm{~V}$ ) and arc lamps (NeAr) reflected off of Baade's secondary flat-field screen for pixel response and wavelength calibration. Data were reduced using a combination of IRAF (NOAO onedspec package) and IDL ${ }^{17}$ routines (SpeXtool xcombspec and xtellcor_general; Vacca et al. 2003; Cushing et al. 2004) as described in detail in Burgasser et al. (2010b).

Figure 4 displays the reduced FIRE spectra. Signal to noise at the $1.27 \mu \mathrm{m}$ peak varies from $\sim 20$ for WISE J1812+2721 to $\sim 100$ for WISE J1617+1807. All of the WISE spectra exhibit strong $\mathrm{H}_{2} \mathrm{O}$ and $\mathrm{CH}_{4}$ bands, absorption from the pressurebroadened red wing of the $0.77 \mu \mathrm{m}$ K I doublet, and blue near-infrared spectral energy distributions, characteristic signatures of late-type $\mathrm{T}$ dwarfs. The $1.1 \mu \mathrm{m}$ and $1.6 \mu \mathrm{m}$ bands are particularly deep in the spectra of WISE J1617+1807, WISE J1812+2721, and WISE J2313-8037, comparable to the bands seen in the spectrum of Wolf 940B. These features are weakest in the spectrum of WISE J2359-7335. WISE J2018-7423 exhibits an unusually flat $2.1 \mu \mathrm{m} K$-band flux peak compared to the other sources, a region dominated by collision-induced $\mathrm{H}_{2}$ absorption (Linsky 1969; Saumon et al.

\footnotetext{
17 Interactive Data Language.
}

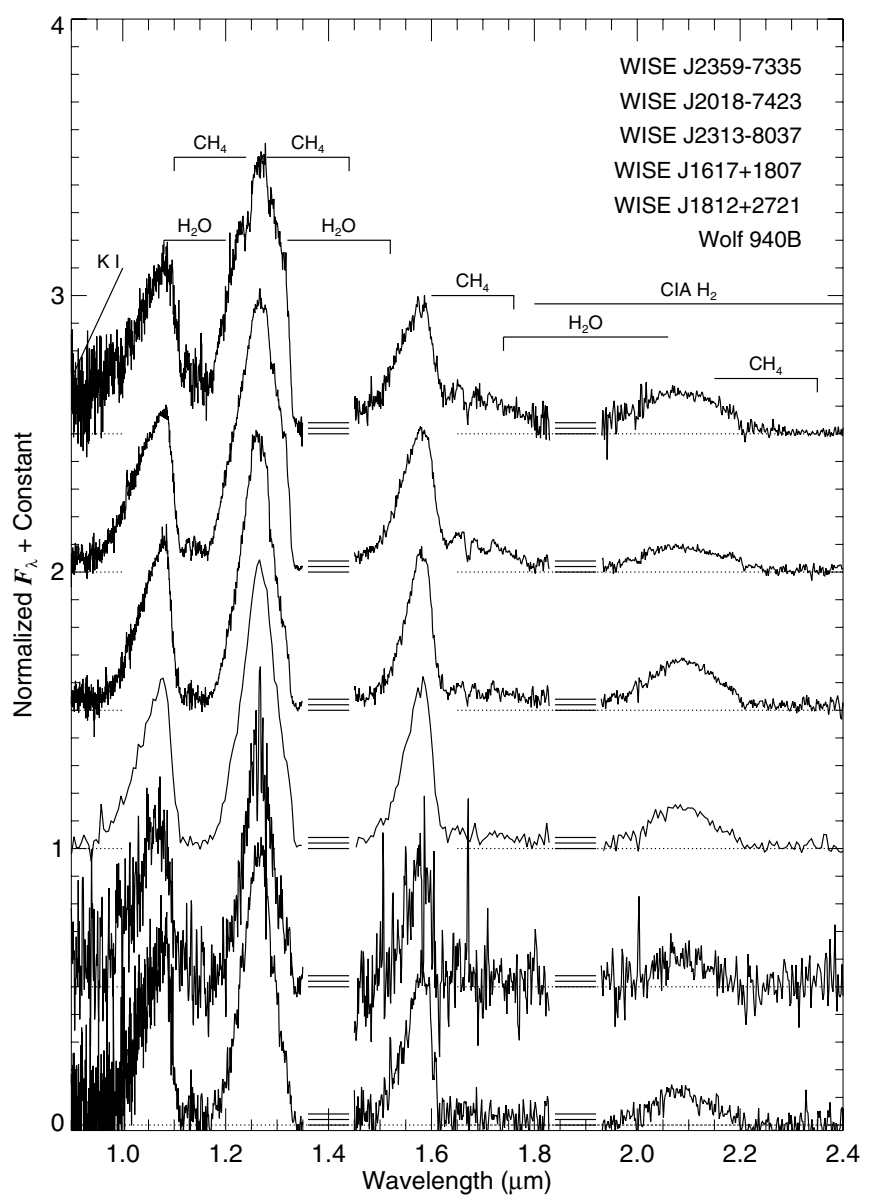

Figure 4. FIRE prism spectra of WISE T dwarf discoveries (in order of spectral morphology) and the T8.5 comparison object Wolf 940B. Spectra are normalized at their $1.3 \mu \mathrm{m}$ spectral peaks and vertically offset by a constant (dotted lines). Regions of strong telluric absorption $(1.35-1.45 \mu \mathrm{m}$ and $1.82-1.95 \mu \mathrm{m})$ have been excised from the plot. Major spectral features characteristic of $\mathrm{T}$ dwarf spectra are labeled.

1994; see Section 6.3). Note that the lower signal-to-noise spectrum of WISE J1812+2721 is due to its faintness $(J=$ $18.19 \pm 0.02)$ and poor observing conditions on 2010 September 19 (airmass $\sim 2$; seeing $\gtrsim 1^{\prime \prime} .5$ ). Nevertheless, its overall spectral shape is clearly indicative of a very late-type $\mathrm{T}$ dwarf. 

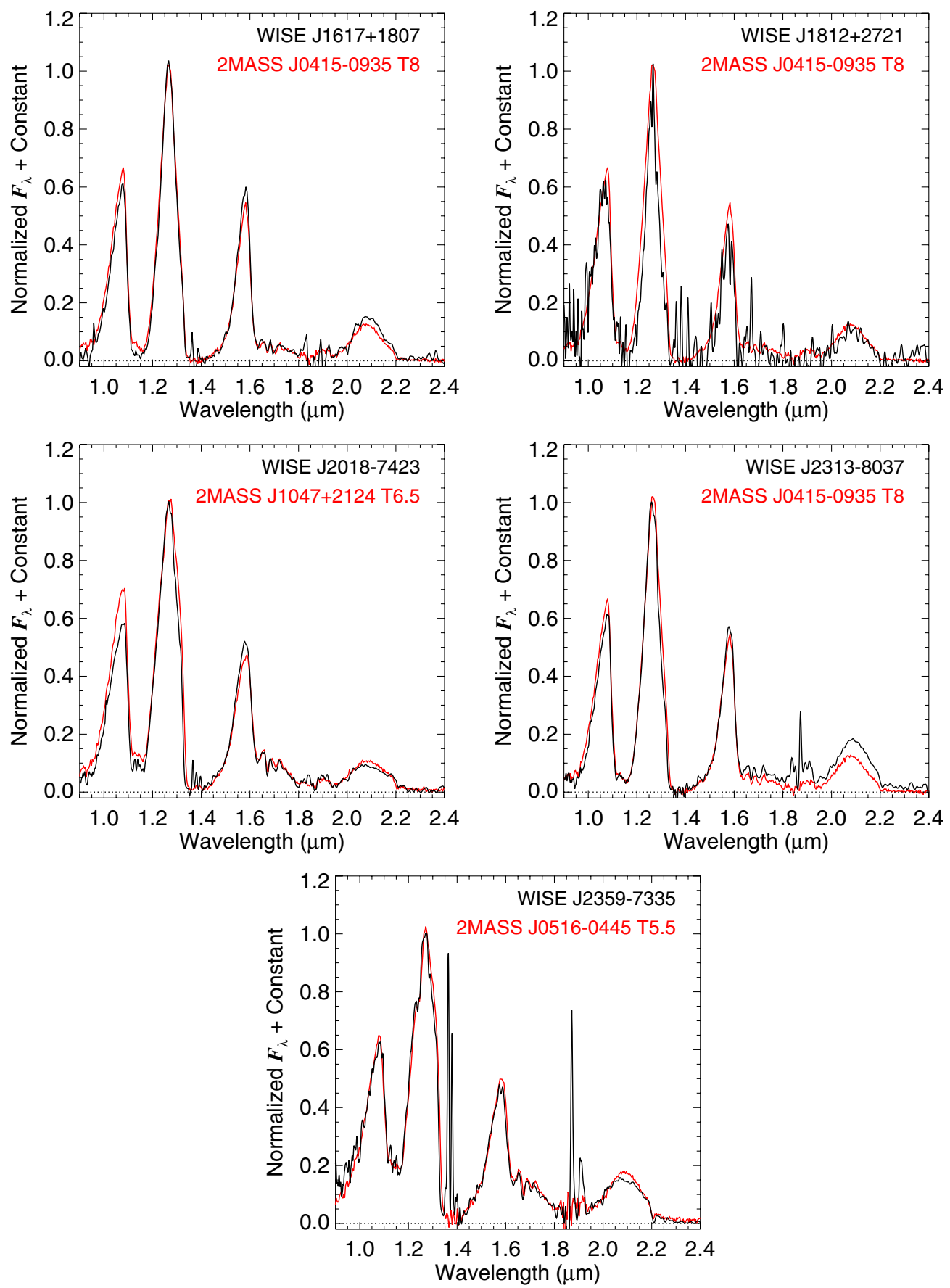

Figure 5. Individual FIRE spectra of the WISE T dwarfs (black lines) compared to their best-fitting SpeX spectral templates (red lines): 2MASS J04151954-0935066 (T8; Burgasser et al. 2002, 2004), 2MASS J10475385+2124234 (T6.5; Burgasser et al. 1999, 2008), and 2MASS J05160945-0445499 (T5.5; Burgasser et al. 2003b, 2008). All spectra are normalized at the $1.3 \mu \mathrm{m}$ flux peaks, and the FIRE data have been smoothed to match the resolution of the SpeX data $(\lambda / \Delta \lambda \approx 120)$ using a Gaussian kernel.

(A color version of this figure is available in the online journal.)

\section{CHARACTERIZING THE T DWARFS}

\subsection{Spectral Classification}

The $\mathrm{T}$ dwarfs were classified using their FIRE spectra following two methods. First, we compared the data to a suite of spectral templates drawn from the SpeX Prism Spectral Libraries, ${ }^{18}$ including the $\mathrm{T}$ dwarf standards defined in Burgasser et al. (2006b). The SpeX prism data (Rayner et al. 2003) have lower resolution than the FIRE data, $\lambda / \Delta \lambda=$

\footnotetext{
18 See http://www.browndwarfs.org/spexprism.
}

90-120; we therefore smoothed the latter to this resolution using a Gaussian kernel. We quantified the agreement between normalized WISE and template spectra using the $\chi^{2}$ statistic, sampling over the wavelength regions $1.0-1.35 \mu \mathrm{m}, 1.45-1.8$ $\mu \mathrm{m}$, and $2.0-2.4 \mu \mathrm{m}$ to avoid strong telluric absorption. Figure 5 displays the best-matching templates for each of the WISE targets. Note that the spectra of WISE J1812+2721 and (to a lesser extent) WISE J2313-8037 appear later than that of the T8 spectral standard 2MASS J04151954-0935066 (hereafter 2MASS J0415-0935; Burgasser et al. 2002) based on their narrower $J$-band flux peaks. 
We also computed the near-infrared classification indices $\mathrm{H}_{2} \mathrm{O}-\mathrm{J}, \mathrm{CH}_{4}-\mathrm{J}, \mathrm{H}_{2} \mathrm{O}-\mathrm{H}, \mathrm{CH}_{4}-\mathrm{H}, \mathrm{CH}_{4}-\mathrm{K}$, and $W_{J}$ from the FIRE data using the definitions given in Burgasser et al. (2006b) and Warren et al. (2007), and the spectral type/index ranges defined in Burningham et al. (2010b) which extend to type T9. For completeness, we also measured the $K / J$ index defined in Burgasser et al. (2006b) and the spectrophotometric $J-K$ color on the $\mathrm{MKO}^{19}$ system following Cushing et al. (2005). We accounted for uncertainty in these measures through Monte Carlo simulation, sampling 1000 realizations of each spectrum varied pixel by pixel by random offsets drawn from a normal distribution scaled to the noise spectrum. The final index values, listed in Table 4, reflect the means and standard deviations of these measurements. The associated spectral types for each index, rounded off to the nearest half subtype, are also listed in Table 4 . These types are generally in agreement with each other and with the template-comparison classification, although the noisier spectrum of WISE $\mathrm{J} 1812+2721$ results in greater scatter. The final classifications were taken as an average of the index and template classifications, accounting for limits in the index types. Classifications range from T5.5 for WISE J2359-7335 to T8.5: for WISE J1812+2721, where the colon indicates an uncertain classification due to noise. WISE J1617+1807, WISE $\mathrm{J} 1812+2721$, and WISE $\mathrm{J} 2313-8037$ are all classified as T8 and later.

\subsection{Estimated Distances and Kinematics}

To estimate the distances of these $\mathrm{T}$ dwarfs, we first derived a linear absolute $W 2$ magnitude/spectral type relation for T6-T8 dwarfs based on WISE photometry (Mainzer et al. 2011) and parallax measurements (Perryman et al. 1997; Tinney et al. 2003; Vrba et al. 2004) for the T6 dwarf SDSSp J162414.37+002915.6 (Strauss et al. 1999), the T7.5 dwarf Gliese 570D (Burgasser et al. 2000), and the T8 dwarf 2MASS J0415-0935. The inferred relation is

$$
M_{W 2}=11.33+0.268 \times S p T,
$$

where $\operatorname{SpT}(\mathrm{T} 6)=6, \operatorname{SpT}(\mathrm{T} 8)=8$, etc. The scatter in the fit is formally $0.03 \mathrm{mag}$; however, due to the small number of calibrators used we assume a systematic uncertainty of 0.1 mag. Distances, taking into account uncertainties in the photometry, spectral classification (0.5-1.0 subtypes), and absolute magnitude relation are listed in Table 5. All of the WISE T dwarfs in this sample are roughly $12-13 \mathrm{pc}$ from the Sun (modulo $1.5-3 \mathrm{pc}$ uncertainties), assuming they are single.

For WISE J2018-7423, WISE J2313-8037, and WISE J2359-7335, we combined these distances with proper motion measurements to infer tangential velocities. We find $V_{\tan }=$ $56 \pm 6 \mathrm{~km} \mathrm{~s}^{-1}, 31 \pm 5 \mathrm{~km} \mathrm{~s}^{-1}$, and $20 \pm 3 \mathrm{~km} \mathrm{~s}^{-1}$ for these sources, respectively. The motions of WISE J2313-8037 and WISE J2359-7335 are consistent with the mean kinematics of nearby field $\mathrm{T}$ dwarfs ( $30 \pm 20 \mathrm{~km} \mathrm{~s}^{-1}$; Faherty et al. 2009), while WISE J2018-7423 is a $\gtrsim 1 \sigma$ outlier. This high velocity source is discussed in further detail in Section 6.3.

\section{SPECTRAL MODEL FITS}

To further characterize these brown dwarfs, we compared our FIRE spectra to both cloudy and cloud-free atmosphere models from Saumon \& Marley (2008). We restricted our analysis to

\footnotetext{
19 Mauna Kea Observatory filter system; see Tokunaga et al. (2002) and Simons \& Tokunaga (2002).
}

the near-infrared spectra alone; i.e., we did not include the WISE photometry. As such, this analysis should be regarded as a preliminary reconnaissance of the atmospheric and physical properties of these dwarfs. A more comprehensive modeling effort will be presented in a forthcoming paper (M. Cushing et al. 2011, in preparation).

We followed a fitting prescription similar to that described in Burgasser et al. (2010b), built upon contemporary work by Cushing et al. (2008) and Bowler et al. (2009). We used solar metallicity models with non-equilibrium chemistry (eddy diffusion parameter $\kappa_{z z}=10^{4} \mathrm{~cm} \mathrm{~s}^{-2}$; Griffith \& Yelle 1999; Saumon et al. 2006; Hubeny \& Burrows 2007) and considered both cloud-free and cloudy models, the latter with condensate sedimentation parameter $f_{\text {sed }}=2$ (Ackerman \& Marley 2001). Atmospheric parameters $T_{\text {eff }}=500-1000 \mathrm{~K}(50 \mathrm{~K}$ steps $)$ and $\log g=4.0-5.5 \mathrm{cgs}$ (0.5 dex steps) were sampled, with corresponding physical parameters (mass, age, and radius) determined using the appropriate evolutionary tracks from Saumon \& Marley (2008). The FIRE spectra were scaled to the apparent $J$-band magnitude of each source, and both models and data were smoothed to a common resolution of $\lambda / \Delta \lambda=$ 300 and sampled at 4 pixels per resolution element to match FIRE's projected slit width. Spectra were compared in the $1.0-1.35 \mu \mathrm{m}, 1.45-1.8 \mu \mathrm{m}$, and $1.95-2.3 \mu \mathrm{m}$ regions, using the $\chi^{2}$ statistic to assess both the goodness of fit and the relative scaling factor $C \equiv(R / d)^{2}$, where $R$ is the radius of the brown dwarf and $d$ is its distance from the Sun. We further constrained our fits by requiring that the model-inferred distance be within $3 \sigma$ of the estimated distance based on $W 2$ photometry (Section 4.2). Note that this constraint is only weakly sensitive to unresolved multiplicity since both distances are based on photometric scaling. Means and uncertainties in the atmospheric parameters were determined using the $F$-distribution probability distribution function $(F-\mathrm{PDF})$ as a weighting factor (Equations (1)-(4) in Burgasser et al. 2010b). Sampling uncertainties of $25 \mathrm{~K}$ and 0.25 dex were also imposed on the inferred $T_{\text {eff }}$ and $\log g$ values, which were propagated into the estimated physical parameters.

Figures 6-11 show the best-fitting models for each of the WISE spectra and for Wolf $940 \mathrm{~B}$, as well as the F-PDF weighted distributions of $T_{\text {eff }}, \log g$, and $f_{\text {sed }}$ parameters. Table 5 summarizes the inferred atmospheric and physical parameters. Overall, the models provide reasonable fits to the spectral data, with the exception of known discrepancies in the core of the 1.6-1.7 $\mu \mathrm{m} \mathrm{CH}_{4}$ band, the strength of the $1.25 \mu \mathrm{m} \mathrm{K} \mathrm{I}$ lines (for the warmer T dwarfs), and the detailed shape of the $1.05 \mu \mathrm{m}$ $Y$-band peak. Fits to WISE J2359-7335 are particularly poor, likely due to the best-fitting models residing at the end of our parameter range. For Wolf 940B, the best-fitting models poorly reproduce the brightness of the observed $K$-band peak. Examining the inferred parameters in detail, we find that $T_{\text {eff }} \mathrm{s}$ track well with spectral type and are consistent with the spectral type $/ T_{\text {eff }}$ scales of Golimowski et al. (2004); Stephens et al. (2009), and Marocco et al. (2010). This correlation may be an artifact of the imposed distance constraints, which are tied to the $M_{W 2} /$ spectral type relation defined above. However, our inferred parameters for Wolf $940 \mathrm{~B}$, which are constrained by the parallactic distance of the system (Harrington \& Dahn 1980), are consistent with the broadband spectral fitting results of Leggett et al. (2010). We infer similar $T_{\text {eff }} \mathrm{s}$ for the WISE targets when the distance constraint is removed. Surprisingly, a range of cloud parameters are indicated, with both WISE J1617+1807 and WISE J2313-8037 exhibiting evidence for the presence of 


\begin{tabular}{|c|c|c|c|c|c|c|}
\hline Index & WISE J1617+1807 & WISE J1812+2721 & WISE J2018-7423 & WISE J2313-8037 & WISE J2359-7335 & Wolf 940B \\
\hline $\mathrm{H}_{2} \mathrm{O}-\mathrm{J}$ & $0.020 \pm 0.004(\geqslant \mathrm{~T} 8)$ & $0.04 \pm 0.05(\mathrm{~T} 7 / \geqslant \mathrm{T} 8)$ & $0.086 \pm 0.006(\mathrm{~T} 7)$ & $0.044 \pm 0.008(\geqslant \mathrm{~T} 8)$ & $0.206 \pm 0.008(\mathrm{~T} 5)$ & $0.026 \pm 0.012(\geqslant \mathrm{~T} 8)$ \\
\hline $\mathrm{CH}_{4}-\mathrm{J}$ & $0.170 \pm 0.003(\geqslant \mathrm{~T} 8)$ & $0.12 \pm 0.03(\geqslant \mathrm{~T} 8)$ & $0.198 \pm 0.004(\geqslant \mathrm{~T} 8)$ & $0.116 \pm 0.004(\geqslant \mathrm{~T} 8)$ & $0.261 \pm 0.004(\mathrm{~T} 7)$ & $0.100 \pm 0.013(\geqslant \mathrm{~T} 8)$ \\
\hline $\mathrm{H}_{2} \mathrm{O}-\mathrm{H}$ & $0.159 \pm 0.007(\mathrm{~T} 8)$ & $0.14 \pm 0.13(\mathrm{~T} 6 / \geqslant \mathrm{T} 9)$ & $0.244 \pm 0.011(\mathrm{~T} 7)$ & $0.174 \pm 0.012(\mathrm{~T} 8)$ & $0.343 \pm 0.011(\mathrm{~T} 5)$ & $0.13 \pm 0.04(\mathrm{~T} 8 / \geqslant \mathrm{T} 9)$ \\
\hline $\mathrm{CH}_{4}-\mathrm{H}$ & $0.108 \pm 0.006(\geqslant \mathrm{~T} 8)$ & $0.29 \pm 0.11(\mathrm{~T} 5 / \mathrm{T} 7)$ & $0.231 \pm 0.010(\mathrm{~T} 7)$ & $0.142 \pm 0.011(\geqslant \mathrm{~T} 8)$ & $0.315 \pm 0.008(\mathrm{~T} 6)$ & $0.08 \pm 0.04(\geqslant \mathrm{~T} 8)$ \\
\hline $\mathrm{CH}_{4}-\mathrm{K}$ & $0.033 \pm 0.016(\geqslant \mathrm{~T} 7)$ & $-0.03 \pm 0.23(\mathrm{~N} / \mathrm{A})$ & $0.17 \pm 0.04(\mathrm{~T} 5 / \geqslant \mathrm{T} 7)$ & $0.14 \pm 0.03(\mathrm{~T} 6 / \geqslant \mathrm{T} 7)$ & $0.145 \pm 0.007(\mathrm{~T} 6)$ & $0.00 \pm 0.12(\mathrm{~N} / \mathrm{A})$ \\
\hline$W_{J}$ & $0.275 \pm 0.003(\mathrm{~T} 8 / \geqslant \mathrm{T} 9)$ & $0.22 \pm 0.04(\geqslant \mathrm{~T} 9)$ & $0.420 \pm 0.005(\leqslant \mathrm{~T} 6)$ & $0.324 \pm 0.005(\mathrm{~T} 8)$ & $0.549 \pm 0.005(\leqslant \mathrm{~T} 6)$ & $0.251 \pm 0.014(\geqslant \mathrm{~T} 9)$ \\
\hline $\mathrm{K} / \mathrm{J}$ & $0.156 \pm 0.002$ & $0.14 \pm 0.03$ & $0.097 \pm 0.003$ & $0.196 \pm 0.004$ & $0.158 \pm 0.002$ & $0.135 \pm 0.012$ \\
\hline$J-K^{\mathrm{a}}$ & $-0.21 \pm 0.05$ & $-0.5 \pm 0.8$ & $-0.54 \pm 0.10$ & $0.12 \pm 0.07$ & $-0.48 \pm 0.02$ & $-0.7 \pm 0.4$ \\
\hline Template SpT & $\mathrm{T} 8$ & $\geqslant \mathrm{~T} 8$ & T6.5 & $\geqslant \mathrm{T} 8$ & $\mathrm{~T} 5.5$ & $\geqslant \mathrm{~T} 8$ \\
\hline Adopted SpT & $\mathrm{T} 8$ & T8.5: & $\mathrm{T} 7$ & $\mathrm{~T} 8$ & T5.5 & $\mathrm{T} 8.5$ \\
\hline
\end{tabular}

Notes. Index spectral types based on the index ranges defined in Burningham et al. (2010b), which incorporates the definitions set out by Burgasser et al. (2006b) for T0-T8 dwarfs and Burningham et al. (2008, for the $W_{J}$ index) for T9 dwarfs. The final type is an average of the index types and the template classification, accounting for upper/lower limits.

${ }^{a}$ Spectrophotometric colors computed from the spectral data following Cushing et al. (2005). 

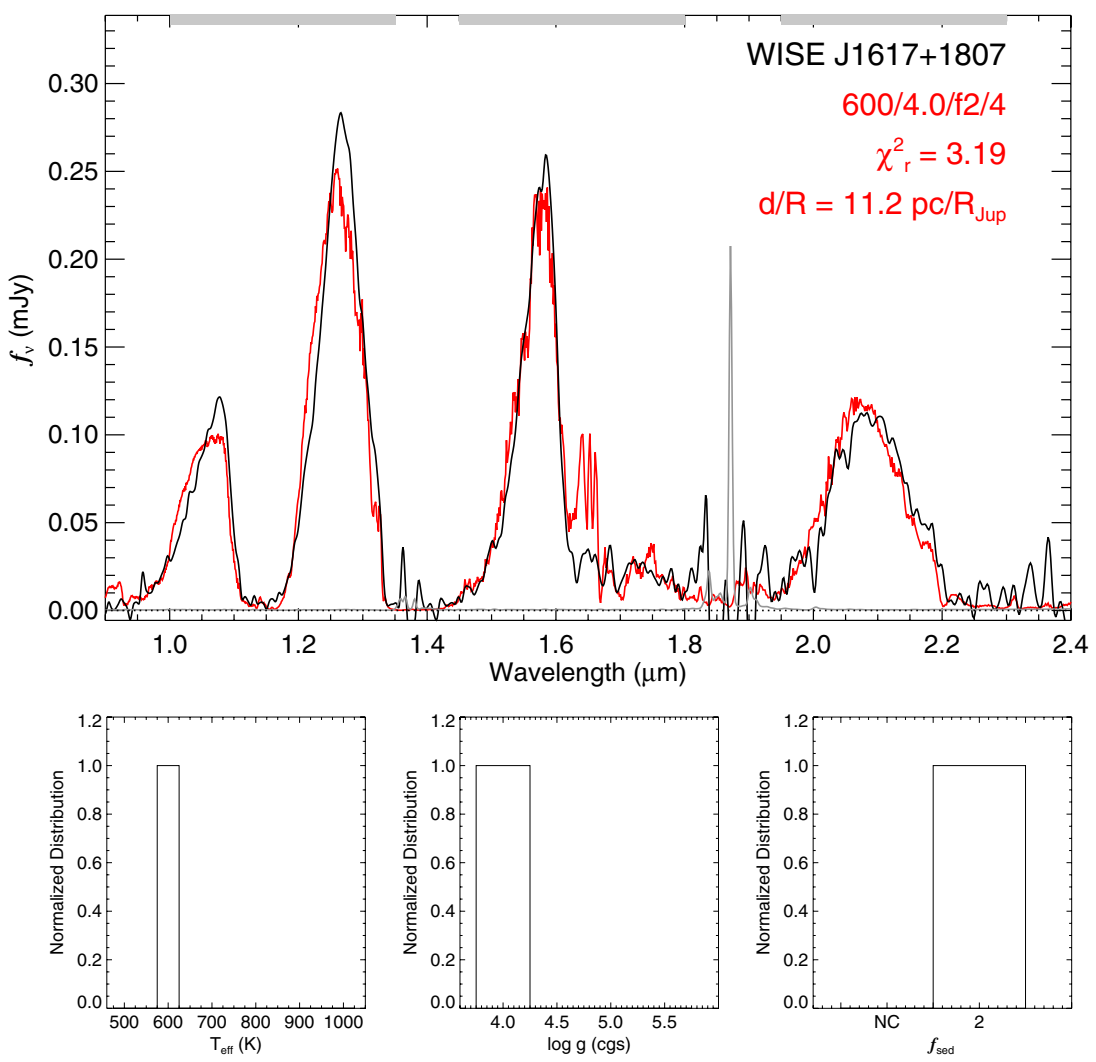

Figure 6. Top panel: best-fitting spectral model (red line) to FIRE data for WISE J1617+1807 (black line). Both spectra are smoothed to the average resolution of the FIRE prism mode $(\lambda / \Delta \lambda \approx 300)$. The data are shown in $f_{v}$ units scaled to the apparent $J$-band magnitude of WISE J1617+1807, and the model scaled to minimize $\chi^{2}$ (the reduced $\chi^{2}$ is listed). Model parameters in the form $T_{\text {eff }} / \log g / f_{\text {sed }} / \log \kappa_{z z}$ are listed, with units as given in the text. We also list the inferred distance-to-radius ratio for this model based on the optimal scaling. Spectral regions over which the fits were made are indicated by the gray bars at top. Bottom panels: from left to right, distributions of $T_{\text {eff }}, \log g$, and $f_{\text {sed }}$ based on an $F$-test PDF factor weighting of each model fit relative to the best-fitting model (see Burgasser et al. 2010b).

(A color version of this figure is available in the online journal.)

Table 5

Results from Model Fits to T Dwarf FIRE Spectra

\begin{tabular}{|c|c|c|c|c|c|c|c|c|}
\hline Source & SpT & $\begin{array}{c}T_{\text {eff }} \\
(\mathrm{K}) \\
\end{array}$ & $\begin{array}{c}\log g \\
\left(\mathrm{~cm} \mathrm{~s}^{-2}\right) \\
\end{array}$ & Cloudy? & $\begin{array}{l}\text { Mass } \\
\left(M_{\mathrm{Jup}}\right) \\
\end{array}$ & $\begin{array}{l}\text { Age } \\
\text { (Gyr) }\end{array}$ & $\begin{array}{c}\text { Model-fit } \\
\text { Distance } \\
(\mathrm{pc})\end{array}$ & $\begin{array}{c}\text { Photometric } \\
\text { Distance } \\
(\mathrm{pc})\end{array}$ \\
\hline WISE J1617+1807 & $\mathrm{T} 8$ & $600_{-30}^{+30}$ & $4.0_{-0.3}^{+0.3}$ & Yes & $7 \pm 3$ & $0.2 \pm 0.3$ & $13.1 \pm 0.6$ & $13.0 \pm 1.5$ \\
\hline WISE J1812+2721 & T8.5: & $620_{-30}^{+30}$ & $4.3_{-0.3}^{+0.3}$ & No & $13 \pm 7$ & $0.9 \pm 1.3$ & $19 \pm 3$ & $13 \pm 3$ \\
\hline WISE J2018-7423 & $\mathrm{T} 7$ & $710_{-60}^{+50}$ & $5.4_{-0.3}^{+0.3}$ & Yes? & $50 \pm 9$ & $10 \pm 4$ & $12.2 \pm 2.3$ & $13.1 \pm 1.4$ \\
\hline WISE J2313-8037 & $\mathrm{T} 8$ & $600_{-30}^{+30}$ & $4.0_{-0.3}^{+0.3}$ & Yes & $7 \pm 3$ & $0.3 \pm 0.4$ & $9.3 \pm 0.4$ & $11.7 \pm 1.6$ \\
\hline WISE J2359-7335 & $\mathrm{T} 5.5$ & $930_{-50}^{+50}$ & $5.1_{-0.4}^{+0.4}$ & No & $38 \pm 18$ & $4 \pm 4$ & $17 \pm 3$ & $12.5 \pm 1.7$ \\
\hline Wolf 940B & $\mathrm{T} 8.5$ & $560_{-30}^{+30}$ & $5.0_{-0.3}^{+0.3}$ & Yes? & $30 \pm 10$ & $7 \pm 4$ & $11.5 \pm 1.6$ & $12.5 \pm 0.7^{a}$ \\
\hline
\end{tabular}

Note. ${ }^{\text {a }}$ Parallax distance measurement for the Wolf 940A primary (Harrington \& Dahn 1980).

photospheric cloud opacity. We discuss some of these secondary parameters in further detail below.

\section{DISCUSSION OF INDIVIDUAL SOURCES}

\subsection{The T8.5: Dwarf WISE J1812+2721}

The latest-type source in this sample is WISE J1812+2721, tentatively classified T8.5: based on spectral comparison to 2MASS J0415-0935 in Figure 5 and spectral indices. It also has the reddest $W 1-W 2$ color in our sample. While this classification is somewhat uncertain due to the lower signal to noise of the spectral data, its binned spectrum is very similar to that of Wolf 940B, itself classified T8.5 (Figure 12). Nearand mid-infrared spectral model fits to Wolf 940B indicate
$T_{\text {eff }}=585-625 \mathrm{~K}$ and $\log g=4.83-5.22$ cgs for an age of 3-10 Gyr (Leggett et al. 2010), similar to the results we infer here. For WISE J1812+2721 we find a comparable $T_{\text {eff }}$ but much lower surface gravity, age, and mass. While surface gravity determinations should in general be treated with caution (see below), the agreement in spectral morphology and inferred $T_{\text {eff }}$ confirms the cool nature of this source.

\subsection{WISE J1617+1807 and WISE J2313-8037: Young and Cloudy-field T Dwarfs?}

WISE J1617+1807 and WISE J2313-8037 exhibit relatively red $J-K$ spectrophotometric colors and large $K / J$ ratios for their spectral types, and our spectral model fits suggest cool $\left(T_{\text {eff }}=600 \mathrm{~K}\right)$, low surface gravity $(\log g=4.0 \mathrm{cgs})$, and 

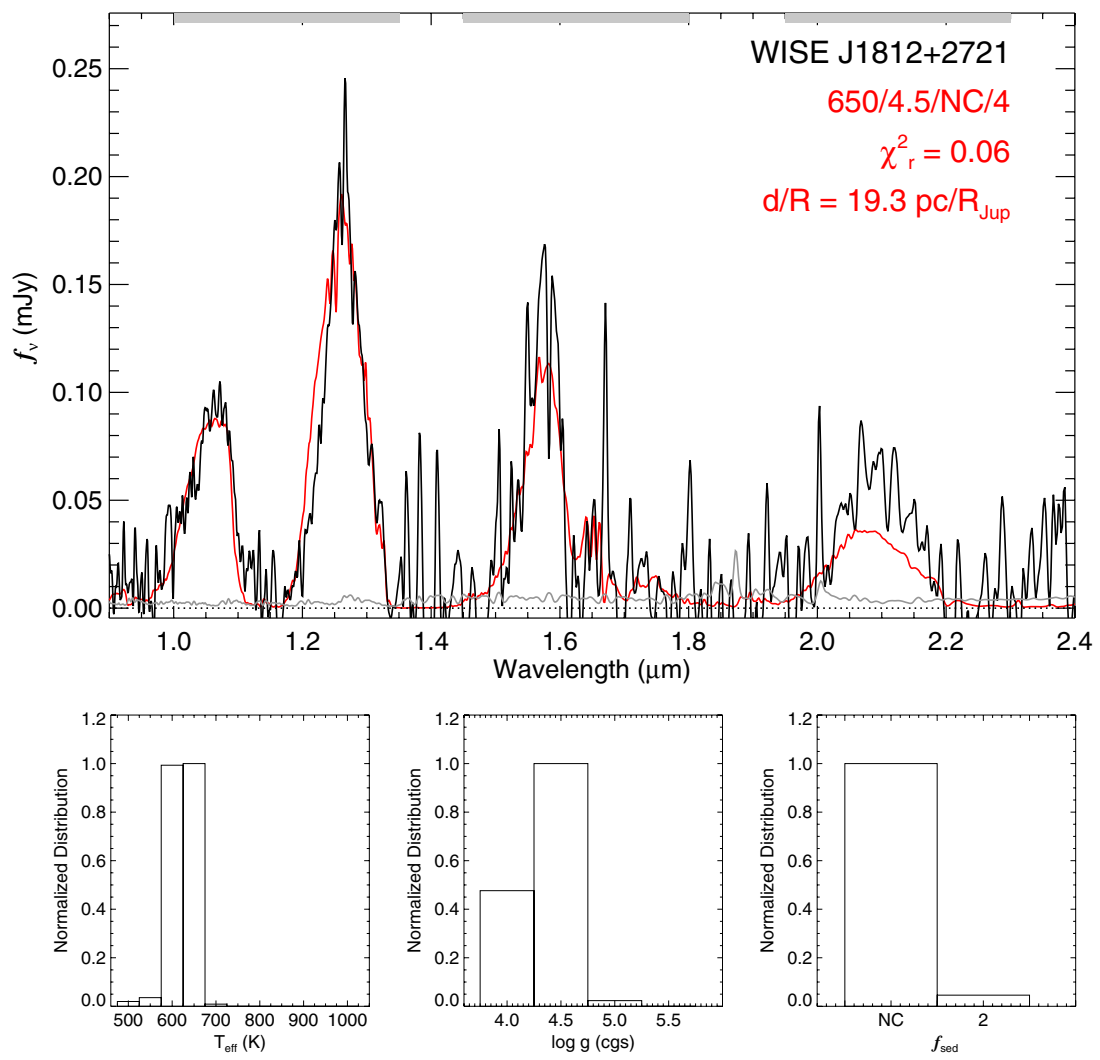

Figure 7. Same as Figure 6 for WISE J1812+2721.

(A color version of this figure is available in the online journal.)
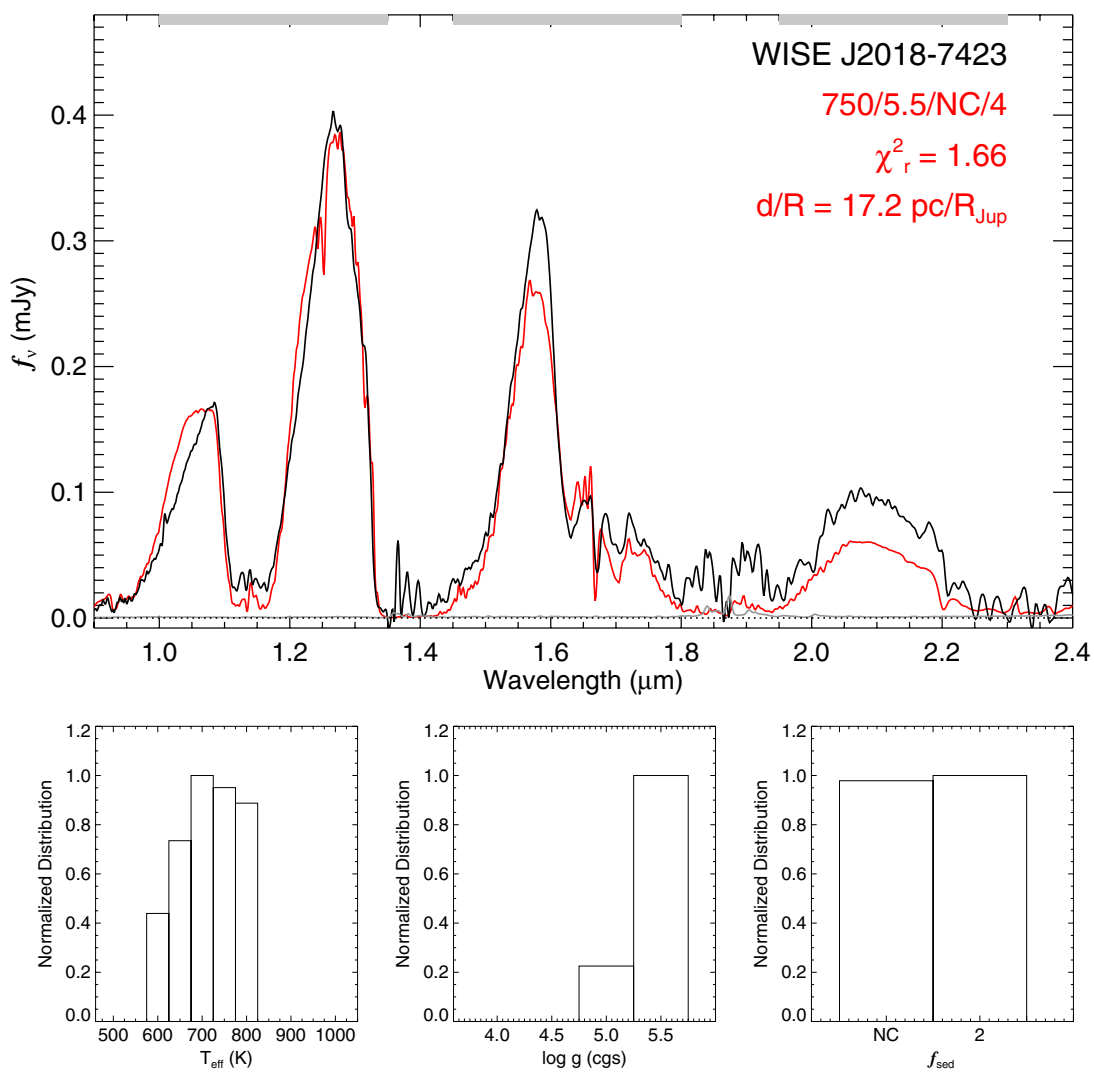

Figure 8. Same as Figure 6 for WISE J2018-7423.

(A color version of this figure is available in the online journal.) 

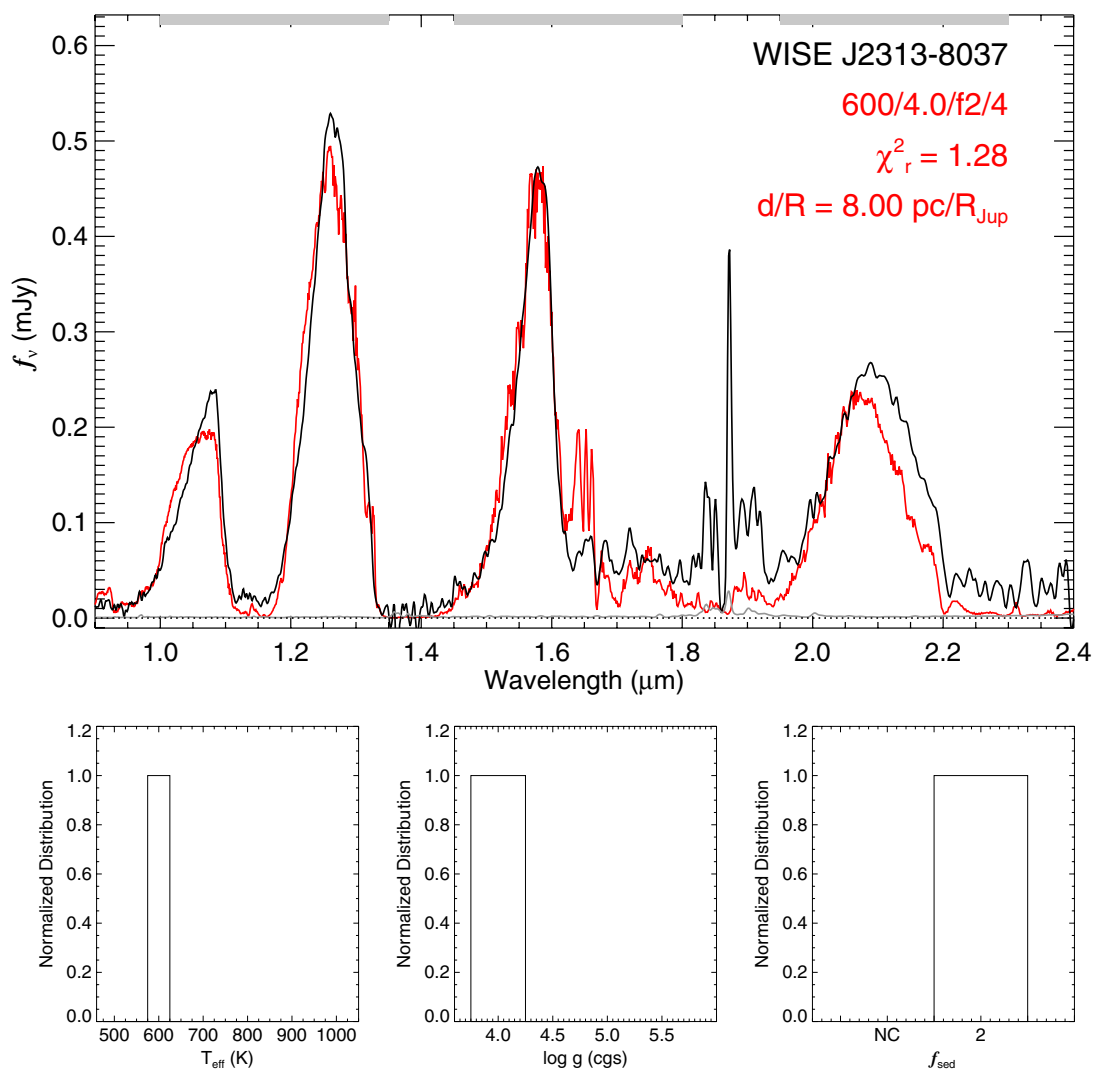

Figure 9. Same as Figure 6 for WISE J2313-8037.

(A color version of this figure is available in the online journal.)
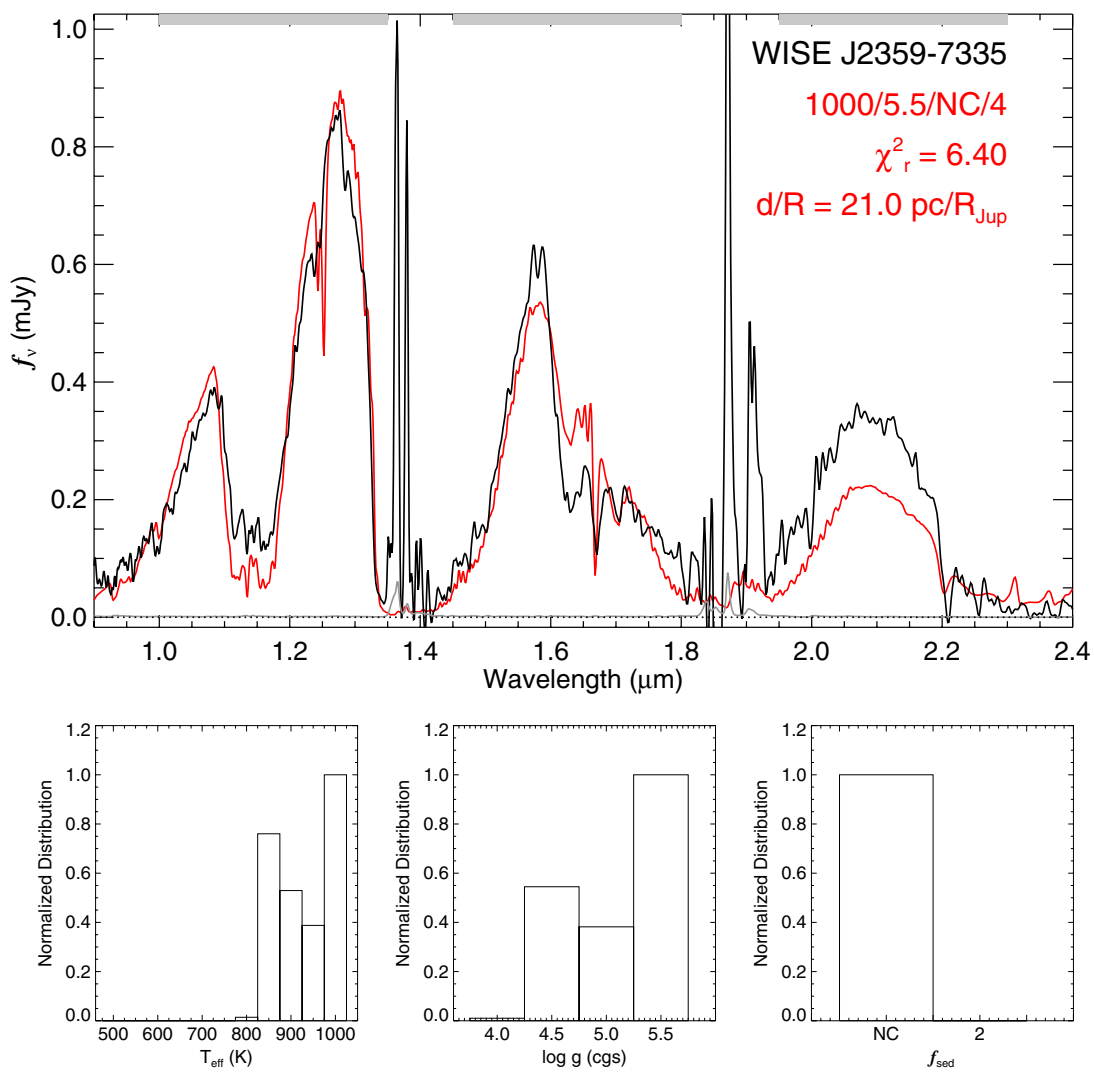

Figure 10. Same as Figure 6 for WISE J2359-7335.

(A color version of this figure is available in the online journal.) 

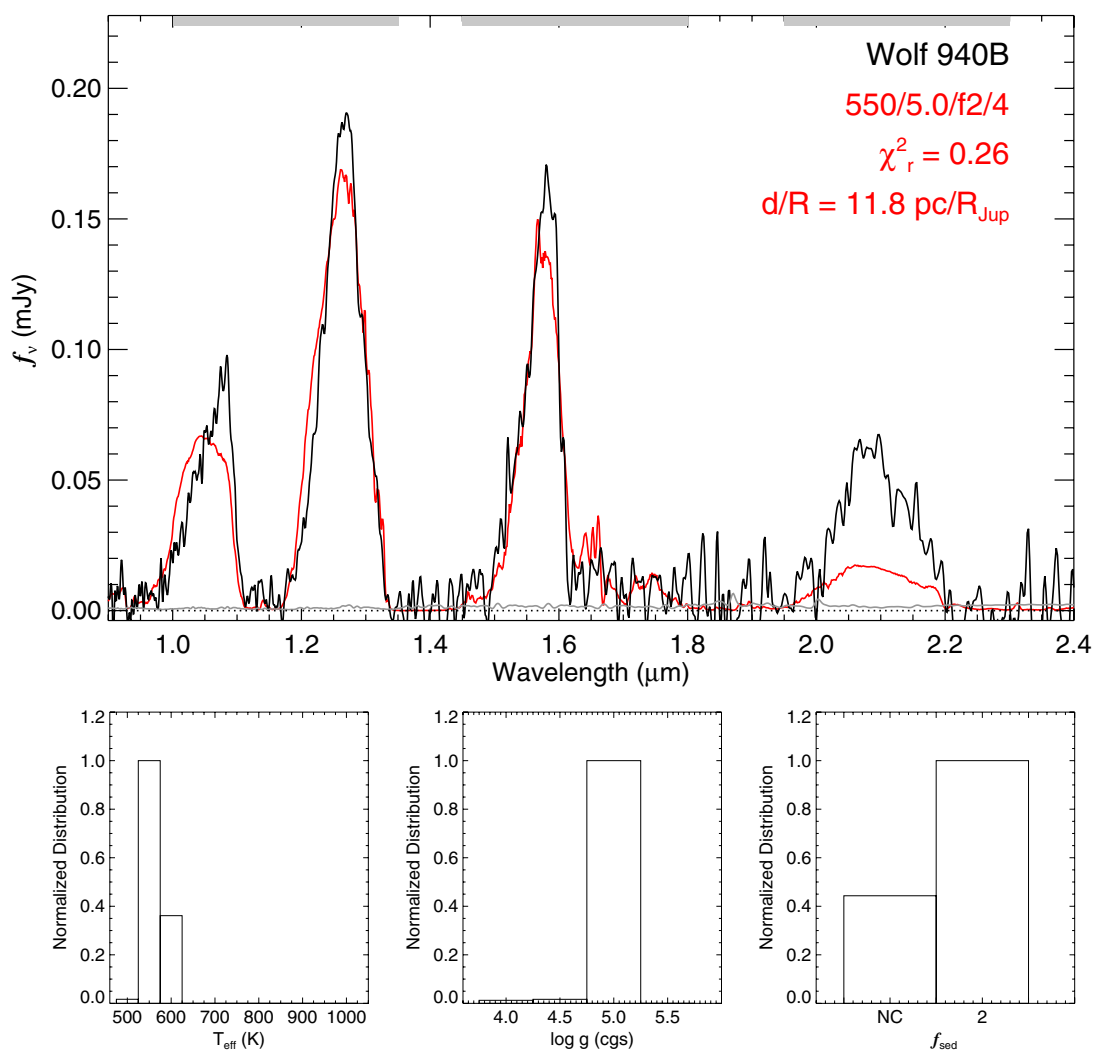

Figure 11. Same as Figure 6 for Wolf 940B.

(A color version of this figure is available in the online journal.)

cloudy atmospheres. The inferred surface gravities are driven largely by the relatively bright $K$-band peaks of these spectra, an indication of reduced collision-induced $\mathrm{H}_{2}$ absorption; surface gravity variations in the absorption strength of this molecule is also cited as an explanation for the red near-infrared colors of young, low-mass L dwarfs (e.g., Lucas et al. 2001; Kirkpatrick et al. 2006, 2008; Allers et al. 2007). Thick clouds may also give rise to reddened $J-K$ colors in L dwarf spectra (e.g., Knapp et al. 2004; Stephens et al. 2009), so it is pertinent that the spectra of both WISE J1617+1807 and WISE J2313-8037 are best fit by cloudy models. Cloud opacity primarily influences the $Y J H$ flux peaks in brown dwarf spectra, which represent minima in gas opacity (Ackerman \& Marley 2001). As such, the models without clouds exhibit $J$-band peaks that are too strong for these two sources. This discrepancy drives our model fits toward cloudier atmospheres.

The presence of clouds has recently been suggested in similar model fits to the T8 dwarf Ross $458 \mathrm{C}$, a widely separated companion to a nearby $\mathrm{M}$ dwarf binary system which has an independent age constraint of 150-800 Myr (Burgasser et al. 2010b; Goldman et al. 2010; Scholz 2010a). The similarity in the inferred properties of Ross 458C, WISE J1617+1807, and WISE J2313-8037-low temperature, low surface gravity, and cloudy atmospheres-appears indicative of a trend toward cloudier atmospheres in younger brown dwarfs. Indeed, such a trend has previously been proposed to explain the spectra of young L dwarfs (e.g., Metchev \& Hillenbrand 2006; Stephens et al. 2009) and in contemporary studies of directly detected exoplanets (e.g., Bowler et al. 2010b; Currie et al. 2011; Madhusudhan et al. 2011). While compelling, evidence for these trends are not yet conclusive. Our model fits for the WISE $\mathrm{T}$ dwarfs indicate relatively young ages

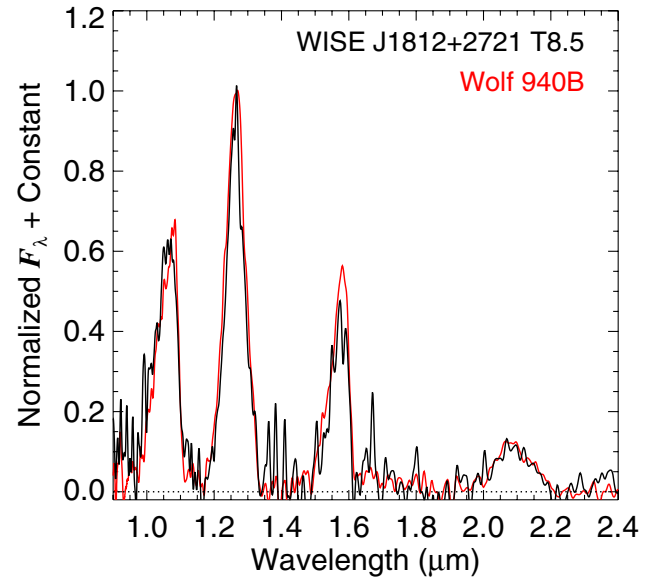

Figure 12. Comparison of FIRE spectra for WISE J1812+2721 (black line) and Wolf $940 \mathrm{~B}$ (red line), both smoothed to a resolution of $\lambda / \Delta \lambda=150$ and normalized at $1.27 \mu \mathrm{m}$.

(A color version of this figure is available in the online journal.)

( $\sim 200-300 \mathrm{Myr})$ and low masses $\left(\sim 7 M_{\text {Jup }}\right)$, values that are somewhat suspect for a pair of isolated field objects (although we cannot rule out membership in a nearby young association such as AB Doradus or Tucana Horologium; Zuckerman et al. 2001, 2004). The fits are also constrained by fairly uncertain spectrophotometric distance estimates. Moreover, we have not considered metallicity variations in this study which are also known to modulate the $K$-band peaks of both $\mathrm{L}$ and $\mathrm{T}$ dwarf spectra (Burgasser et al. 2006a; Burgasser 2007; Liu et al. 2007; Looper et al. 2008). We therefore regard the increased role of clouds in shaping young $\mathrm{T}$ dwarf spectra as a suggestive trend 


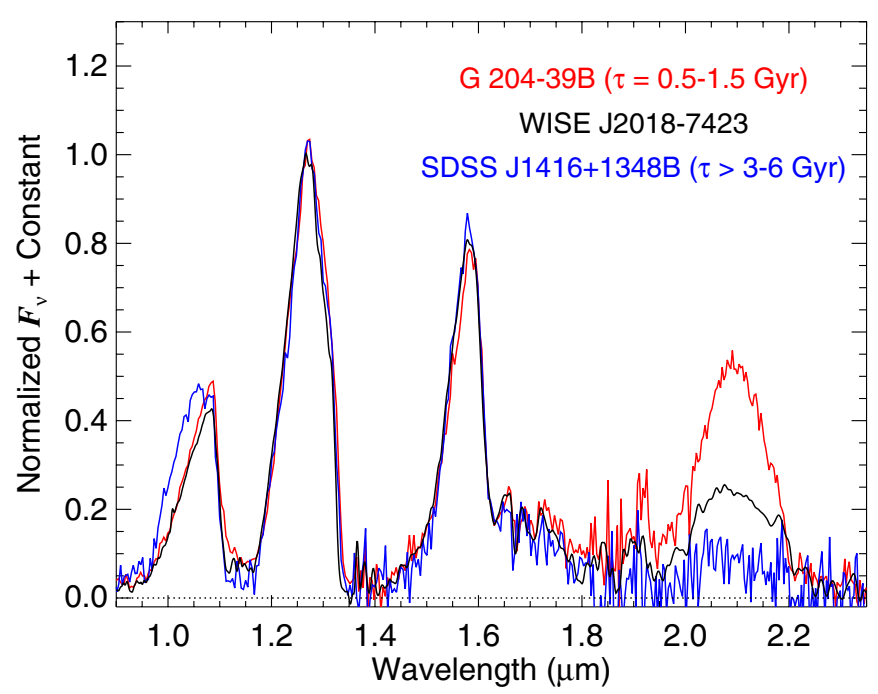

Figure 13. Comparison of the near-infrared spectra of WISE J2018-7423 (black line; FIRE data) and the T dwarf companions G 204-39B (red line; SpeX data from Burgasser et al. 2006a) and SDSS J1416+1348B (blue line; SpeX data from Burgasser et al. 2010a). All three spectra are normalized at their $1.27 \mu \mathrm{m}$ spectral peaks.

(A color version of this figure is available in the online journal.)

and defer further analysis to more comprehensive, broadband spectral modeling (M. Cushing 2011, in preparation).

\subsection{WISEPC J201824.98-742326.1: An Old Blue T Dwarf?}

WISE J2018-7423 exhibits an opposing spectral peculiarity: a suppressed $K$-band peak resulting in an unually blue spectrophotometric near-infrared color $(J-K=-0.54 \pm$ $0.10)$ and small $K / J$ index $(0.097 \pm 0.003)$ for its spectral type. Previously identified blue $\mathrm{T}$ dwarfs, such as $2 \mathrm{MASS}$ J09373487+2931409 $(J-K=-1.10 \pm 0.07 ; K / J=$ 0.08; Burgasser et al. 2002, 2006a; Knapp et al. 2004) and SDSS J141624.08+134826.7B (hereafter SDSS J1416+1348B, $J-K=-1.58 \pm 0.17 ; K / J=0.037 \pm 0.004$; Burningham et al. 2010a; Burgasser et al. 2010a; Scholz 2010b), have similarly suppressed $K$-band peaks from strong collision-induced $\mathrm{H}_{2}$ absorption, attributed to a high surface gravity and/or subsolar metallicity. Our spectral model fits support a high surface gravity for this source, indicating $\log g \sim 5.4 \mathrm{cgs}$, age $\tau \gtrsim 6 \mathrm{Gyr}$ and mass $\mathrm{M} \sim 50 M_{\mathrm{Jup}}$; the estimated $V_{\tan }=56 \pm 6 \mathrm{~km} \mathrm{~s}^{-1}$ of this object supports a relatively old age. However, our fits cannot test whether this source is metal-poor.

Fortunately, metallicity effects can be separately discerned in the $1.05 \mu \mathrm{m} Y$-band peak, which is broadened in both the theoretical and observed spectra of metal-poor T dwarfs (Burgasser et al. 2006a, 2010a). In Figure 13 we compare the spectrum of WISE J2018-7423 to those of two equivalently classified $\mathrm{T}$ dwarf companions to stars with independent age and metallicity constraints: the young, metal-rich T6.5 G 204-39B (a.k.a. SDSS J175805.46+463311.9; Knapp et al. 2004; Faherty et al. 2010) and the old, metal-poor T7 SDSS J1416+1348B. G 204-39A is an M3 star which exhibits weak signatures of $\mathrm{H} \alpha$ and X-ray activity consistent with $\tau=$ $0.5-1.5 \mathrm{Gyr}$, and optical spectral indicators (i.e., ratio of $\mathrm{TiO} / \mathrm{CaH}$ ) suggesting a slightly supersolar metallicity. SDSS $\mathrm{J} 1416+1348 \mathrm{~A}$ is an unusually blue L dwarf, and spectral model fits to both primary and secondary indicate an older ( $\tau>3 \mathrm{Gyr})$ and possibly metal-poor system (e.g., Bowler et al. 2010a; Burgasser et al. 2010a, 2010b; Cushing et al. 2010. As Figure 13 shows, the spectra of all three sources are roughly equivalent in the $1.2-1.8 \mu \mathrm{m}$ region, but vary in $K$-band peak brightness, with WISE J2018-7423 being the intermediate source. More importantly, WISE J2018-7423 does not have the broadened $1.05 \mu \mathrm{m}$ peak seen in the spectrum of SDSS J1416+1348B. This comparison suggests that WISE J2018-7423 is a roughly solar-metallicity field brown dwarf that is both older and more massive than the average local population.

\section{SUMMARY}

We have identified five new late-type T dwarfs with WISE, confirmed through low-resolution, near-infrared spectroscopy with the Magellan FIRE spectrograph. The spectra indicate classifications ranging from T5.5 to T8.5: with the latest-type source, WISE J1812+2721, found to be an excellent match to the T8.5 companion brown dwarf Wolf 940B. Estimated distances are roughly $12-13 \mathrm{pc}$, assuming single sources. Preliminary spectral model fits indicate $T_{\text {eff }} \mathrm{S}$ as low as $600 \mathrm{~K}$, with a broad range of surface gravities, masses, ages, and cloud properties. In particular, WISE J1617+1807 and WISE J2313-8037 show indications of being young, low-mass, and cloudy based on the relative strengths of their $J H K$ flux peaks, characteristics similar to the 150-800 Myr T8 companion Ross 458C; while the relatively blue and high proper motion T dwarf WISE J2018-7423 may be a solar-metallicity, older, and more massive brown dwarf. Validation of the atmospheric and physical properties of these objects requires more comprehensive broadband modeling with improved treatment of molecular opacities and the role of clouds. Nevertheless, it is clear from these early results that WISE will produce an extensive and diverse sample of cool brown dwarfs that can be used to improve our physical understanding of low-temperature, substellar atmospheres (J. D. Kirkpatrick et al. 2011, in preparation).

The authors thank telescope operators Mauricio Martinez, Sergio Vara, and Jorge Araya at Magellan for their assistance with the FIRE and LDSS-3 observations, and T. Jarrett for providing scripts and guidance for the WIRC imaging data reduction. A.J.B. acknowledges financial support from the Chris and Warren Hellman Fellowship Program. This publication makes use of data products from the Wide-field Infrared Survey Explorer, which is a joint project of the University of California, Los Angeles, and the Jet Propulsion Laboratory/California Institute of Technology, funded by the National Aeronautics and Space Administration. This publication also makes use of data products from NEOWISE, which is a project of the Jet Propulsion Laboratory/California Institute of Technology, funded by the Planetary Science Division of the National Aeronautics and Space Administration. This publication makes use of data from the Two Micron All Sky Survey, which is a joint project of the University of Massachusetts and the Infrared Processing and Analysis Center, and funded by the National Aeronautics and Space Administration and the National Science Foundation. 2MASS data were obtained from the NASA/IPAC Infrared Science Archive, which is operated by the Jet Propulsion Laboratory, California Institute of Technology, under contract with the National Aeronautics and Space Administration. The Digitized Sky Surveys were produced at the Space Telescope Science Institute under U.S. Government grant NAG W-2166. The images of these surveys are based on photographic data obtained using the Oschin Schmidt Telescope on Palomar Mountain and the UK Schmidt Telescope. The Second Palomar Observatory Sky Survey (POSS-II) was made by the California Institute 
of Technology with funds from the National Science Foundation, the National Geographic Society, the Sloan Foundation, the Samuel Oschin Foundation, and the Eastman Kodak Corporation. The Oschin Schmidt Telescope is operated by the California Institute of Technology and Palomar Observatory. This research has also made use of the SIMBAD database, operated at CDS, Strasbourg, France; the M, L, and T dwarf compendium housed at http://DwarfArchives.org and maintained by Chris Gelino, Davy Kirkpatrick, and Adam Burgasser; and the SpeX Prism Spectral Libraries, maintained by Adam Burgasser at http://www.browndwarfs.org/spexprism.

Facilities: AAT (IRIS2), FMO:31in (FANCAM), Magellan: Baade (FIRE), Magellan: Clay (LDSS-3), Hale (WIRC), SOAR (SpartanIRC)

\section{REFERENCES}

Ackerman, A. S., \& Marley, M. S. 2001, ApJ, 556, 872

Allers, K. N., et al. 2007, ApJ, 657, 511

Allington-Smith, J., et al. 1994, PASP, 106, 983

Bowler, B. P., Liu, M. C., \& Cushing, M. C. 2009, ApJ, 706, 1114

Bowler, B. P., Liu, M. C., \& Dupuy, T. J. 2010a, ApJ, 710, 45

Bowler, B. P., Liu, M. C., Dupuy, T. J., \& Cushing, M. C. 2010b, ApJ, 723, 850

Burgasser, A. J. 2004, ApJS, 155, 191

Burgasser, A. J. 2007, ApJ, 658, 617

Burgasser, A. J., Burrows, A., \& Kirkpatrick, J. D. 2006a, ApJ, 639, 1095

Burgasser, A. J., Cruz, K. L., \& Kirkpatrick, J. D. 2007, ApJ, 657, 494

Burgasser, A. J., Geballe, T. R., Leggett, S. K., Kirkpatrick, J. D., \& Golimowski, D. A. 2006b, ApJ, 637, 1067

Burgasser, A. J., Kirkpatrick, J. D., Liebert, J., \& Burrows, A. 2003a, ApJ, 594, 510

Burgasser, A. J., Liu, M. C., Ireland, M. J., Cruz, K. L., \& Dupuy, T. J. 2008, ApJ, 681,579

Burgasser, A. J., Looper, D., \& Rayner, J. T. 2010a, AJ, 139, 2448

Burgasser, A. J., McElwain, M. W., \& Kirkpatrick, J. D. 2003b, AJ, 126, 2487

Burgasser, A. J., McElwain, M. W., Kirkpatrick, J. D., Cruz, K. L., Tinney,

C. G., \& Reid, I. N. 2004, AJ, 127, 2856

Burgasser, A. J., et al. 1999, ApJ, 522, L65

Burgasser, A. J., et al. 2000, ApJ, 531, L57

Burgasser, A. J., et al. 2002, ApJ, 564, 421

Burgasser, A. J., et al. 2010b, ApJ, 725, 1405

Burningham, B., et al. 2008, MNRAS, 391, 320

Burningham, B., et al. 2009, MNRAS, 395, 1237

Burningham, B., et al. 2010a, MNRAS, 404, 1952

Burningham, B., et al. 2010b, MNRAS, 406, 1885

Burrows, A., Sudarsky, D., \& Lunine, J. I. 2003, ApJ, 596, 587

Chiu, K., Fan, X., Leggett, S. K., Golimowski, D. A., Zheng, W., Geballe, T. R., Schneider, D. P., \& Brinkmann, J. 2006, AJ, 131, 2722

Currie, T., Bailey, V., Fabrycky, D., Murray-Clay, R., Rodigas, T., \& Hinz, P. 2010, ApJ, 721, L177

Currie, T., et al. 2011, ApJ, 729, 128

Cushing, M. C., Rayner, J. T., \& Vacca, W. D. 2005, ApJ, 623, 1115

Cushing, M. C., Saumon, D., \& Marley, M. S. 2010, AJ, 140, 1428

Cushing, M. C., Vacca, W. D., \& Rayner, J. T. 2004, PASP, 116, 362

Cushing, M. C., et al. 2008, ApJ, 678, 1372

Delorme, P., et al. 2008a, A\&A, 482, 961

Delorme, P., et al. 2008b, A\&A, 484, 469

Faherty, J. K., Burgasser, A. J., Cruz, K. L., Shara, M. M., Walter, F. M., \& Gelino, C. R. 2009, AJ, 137, 1

Faherty, J. K., Burgasser, A. J., West, A. A., Bochanski, J. J., Cruz, K. L., Shara, M. M., \& Walter, F. M. 2010, AJ, 139, 176

Geballe, T. R., et al. 2002, ApJ, 564, 466

Gelino, C., et al. 2011, AJ, submitted

Goldman, B., Marsat, S., Henning, T., Clemens, C., \& Greiner, J. 2010, MNRAS, 405,1140

Golimowski, D. A., et al. 2004, AJ, 127, 3516

Graff, D. S., \& Freese, K. 1996, ApJ, 467, L65

Griffith, C. A., \& Yelle, R. V. 1999, ApJ, 519, L85

Hamuy, M., et al. 2006, PASP, 118, 2

Harrington, R. S., \& Dahn, C. C. 1980, AJ, 85, 454

Hubeny, I., \& Burrows, A. 2007, ApJ, 669, 1248
Janson, M., Bergfors, C., Goto, M., Brandner, W., \& Lafrenière, D. 2010, ApJ, 710, L35

Kalas, P., et al. 2008, Science, 322, 1345

Kanneganti, S., Park, C., Skrutskie, M. F., Wilson, J. C., Nelson, M. J., Smith, A. W., \& Lam, C. R. 2009, PASP, 121, 885

Kirkpatrick, J. D. 2005, ARA\&A, 43, 195

Kirkpatrick, J. D., Barman, T. S., Burgasser, A. J., McGovern, M. R., McLean, I. S., Tinney, C. G., \& Lowrance, P. J. 2006, ApJ, 639, 1120

Kirkpatrick, J. D., et al. 2008, ApJ, 689, 1295

Knapp, G. R., et al. 2004, AJ, 127, 3553

Lawrence, A., et al. 2007, MNRAS, 379, 1599

Leggett, S. K., Marley, M. S., Freedman, R., Saumon, D., Liu, M. C., Geballe, T. R., Golimowski, D. A., \& Stephens, D. C. 2007, ApJ, 667, 537

Leggett, S. K., Saumon, D., Burningham, B., Cushing, M. C., Marley, M. S., \& Pinfield, D. J. 2010, ApJ, 720, 252

Linsky, J. L. 1969, ApJ, 156, 989

Liu, M. C., Leggett, S. K., \& Chiu, K. 2007, ApJ, 660, 1507

Liu, M. C., et al. 2011, arXiv:1103.0014

Lodders, K. 1999, ApJ, 519, 793

Lodders, K., \& Fegley, B. 2002, Icarus, 155, 393

Lodieu, N., et al. 2007, MNRAS, 379, 1423

Loh, E. D., Biel, J. D., Chen, J., Davis, M., Laporte, R., \& Loh, O. Y. 2004, Proc. SPIE, 5492, 1644

Looper, D. L., Kirkpatrick, J. D., \& Burgasser, A. J. 2007, AJ, 134, 1162

Looper, D. L., et al. 2008, ApJ, 686, 528

Lucas, P. W., Roche, P. F., Allard, F., \& Hauschildt, P. H. 2001, MNRAS, 326 , 695

Lucas, P. W., et al. 2010, MNRAS, 408, L56

Luhman, K. L., Burgasser, A. J., \& Bochanski, J. J. 2011, ApJ, 730, L9

Madhusudhan, N., Burrows, A., \& Currie, T. 2011, arXiv:1102.5089

Mainzer, A., et al. 2011, ApJ, 726, 30

Marley, M. S., Seager, S., Saumon, D., Lodders, K., Ackerman, A. S., Freedman, R. S., \& Fan, X. 2002, ApJ, 568, 335

Marocco, F., et al. 2010, A\&A, 524, A38

Marois, C., Macintosh, B., Barman, T., Zuckerman, B., Song, I., Patience, J., Lafrenière, D., \& Doyon, R. 2008, Science, 322, 1348

Metchev, S. A., \& Hillenbrand, L. A. 2006, ApJ, 651, 1166

Nakajima, T., Oppenheimer, B. R., Kulkarni, S. R., Golimowski, D. A., Matthews, K., \& Durrance, S. T. 1995, Nature, 378, 463

Oke, J. B., et al. 1995, PASP, 107, 375

Oppenheimer, B. R., Kulkarni, S. R., Matthews, K., \& Nakajima, T. 1995, Science, 270, 1478

Oppenheimer, B. R., Kulkarni, S. R., Matthews, K., \& van Kerkwijk, M. H. 1998, ApJ, 502, 932

Perryman, M. A. C., et al. 1997, A\&A, 323, L49

Rayner, J. T., Toomey, D. W., Onaka, P. M., Denault, A. J., Stahlberger, W. E., Vacca, W. D., Cushing, M. C., \& Wang, S. 2003, PASP, 115, 362

Reylé, C., et al. 2010, A\&A, 522, A112

Saumon, D., Bergeron, P., Lunine, J. I., Hubbard, W. B., \& Burrows, A. 1994, ApJ, 424, 333

Saumon, D., \& Marley, M. S. 2008, ApJ, 689, 1327

Saumon, D., Marley, M. S., Cushing, M. C., Leggett, S. K., Roellig, T. L., Lodders, K., \& Freedman, R. S. 2006, ApJ, 647, 552

Scholz, R. 2010a, A\&A, 515, A92

Scholz, R. 2010b, A\&A, 510, L8

Simcoe, R. A., et al. 2008, Proc. SPIE, 7014, 70140U

Simcoe, R. A., et al. 2010, Proc. SPIE, 7735, 773514

Simons, D. A., \& Tokunaga, A. 2002, PASP, 114, 169

Skrutskie, M. F., et al. 2006, AJ, 131, 1163

Stephens, D. C., et al. 2009, ApJ, 702, 154

Strauss, M. A., et al. 1999, ApJ, 522, L61

Tinney, C. G., Burgasser, A. J., \& Kirkpatrick, J. D. 2003, AJ, 126, 975

Tinney, C. G., Burgasser, A. J., Kirkpatrick, J. D., \& McElwain, M. W. 2005, AJ, 130,2326

Tinney, C. G., et al. 2004, Proc. SPIE, 5492, 998

Tody, D. 1986, Proc. SPIE, 627, 733

Tokunaga, A. T., Simons, D. A., \& Vacca, W. D. 2002, PASP, 114, 180

Vacca, W. D., Cushing, M. C., \& Rayner, J. T. 2003, PASP, 115, 389

Vrba, F. J., et al. 2004, AJ, 127, 2948

Warren, S. J., et al. 2007, MNRAS, 381, 1400

Whitworth, A. P., \& Stamatellos, D. 2006, A\&A, 458, 817

Wilson, J. C., et al. 2003, Proc. SPIE, 4841, 451

Wright, E. L., et al. 2010, AJ, 140, 1868

York, D. G., et al. 2000, AJ, 120, 1579

Zacharias, N., et al. 2010, AJ, 139, 2184

Zuckerman, B., Song, I., \& Bessell, M. S. 2004, ApJ, 613, L65

Zuckerman, B., Song, I., \& Webb, R. A. 2001, ApJ, 559, 388 